\title{
Nonemptiness and smoothness of twisted Brill-Noether loci
}

\author{
George H. Hitching ${ }^{1} \cdot$ Michael Hoff ${ }^{2}$ (D) . Peter E. Newstead ${ }^{3}$
}

Received: 19 July 2019 / Accepted: 1 June 2020 / Published online: 25 June 2020

(c) The Author(s) 2020

\begin{abstract}
Let $V$ be a vector bundle over a smooth curve $C$. In this paper, we study twisted BrillNoether loci parametrising stable bundles $E$ of rank $n$ and degree $e$ with the property that $h^{0}(C, V \otimes E) \geq k$. We prove that, under conditions similar to those of Teixidor i Bigas and of Mercat, the Brill-Noether loci are nonempty and in many cases have a component which is generically smooth and of the expected dimension. Along the way, we prove the irreducibility of certain components of both twisted and "nontwisted" Brill-Noether loci. We describe the tangent cones to the twisted Brill-Noether loci. We end with an example of a general bundle over a general curve having positive-dimensional twisted Brill-Noether loci with negative expected dimension.
\end{abstract}

Keywords Brill-Noether loci $\cdot$ Petri trace map $\cdot$ Vector bundles

Mathematics Subject Classification $14 \mathrm{H} 60 \cdot 14 \mathrm{H} 51$

\section{Introduction}

Let $C$ be a smooth projective curve over an algebraically closed field $\mathbb{K}$ of characteristic zero. A fundamental feature of the geometry of $C$ and $\operatorname{Pic}^{d}(C)$ is the Brill-Noether locus

$$
W_{d}^{r}(C)=\left\{L \in \operatorname{Pic}^{d}(C): h^{0}(C, L) \geq r+1\right\} .
$$

These objects have been much studied. The expected dimension of $W_{d}^{r}(C)$ is the Brill-Noether number $\rho(g, d, r)=g-(r+1)(g-d+r)$ where $g$ is the genus of $C$; every

Michael Hoff

hahn@math.uni-sb.de

George H. Hitching

gehahi@oslomet.no

Peter E. Newstead

newstead@liv.ac.uk

1 Oslo Metropolitan University, Postboks 4, St. Olavs plass, 0130 Oslo, Norway

2 Universität des Saarlandes, Campus E2 4, D-66123 Saarbrücken, Germany

3 Department of Mathematical Sciences, University of Liverpool, Peach Street, Liverpool L69 7ZL, UK 
irreducible component has dimension $\geq \rho(g, d, r)$, and a great deal is known about these loci (for details, see Sect. 2).

A natural generalisation of (1.1) to vector bundles of higher rank is given as follows. We denote by $U^{s}(n, e)$ the moduli space of stable bundles of rank $n$ and degree $e$ over $C$. This is an irreducible quasiprojective variety of dimension $n^{2}(g-1)+1$. The generalised Brill-Noether locus $B_{n, e}^{k}$ is defined set-theoretically by

$$
B_{n, e}^{k}=\left\{E \in U^{s}(n, e): h^{0}(C, E) \geq k\right\} .
$$

(In this notation, $W_{d}^{r}(C)$ is written $B_{1, d}^{r+1}$.) These loci have also been studied in much detail, although the results for the case $n=1$ do not necessarily generalise. Brill-Noether loci are also closely related to moduli of coherent systems, that is, pairs $(V, \Lambda)$ where $V$ is a vector bundle and $\Lambda$ a subspace of $H^{0}(C, V)$ of a fixed dimension.

In the present work we study another generalisation of $B_{n, e}^{k}$, which to our knowledge was first defined in [36, Sect. 2]. Fix a vector bundle $V$ over $C$ of rank $r$ and degree $d$ (not necessarily semistable). Then the twisted Brill-Noether locus $B_{n, e}^{k}(V)$ is defined set-theoretically by

$$
B_{n, e}^{k}(V):=\left\{E \in U^{s}(n, e): h^{0}(C, V \otimes E) \geq k\right\} .
$$

The space $U^{s}(n, e)$ is an open subset of the moduli space $U(n, e)$ of S-equivalence classes of semistable bundles of rank $n$ and degree $e$. We write $[E]$ for the S-equivalence class of a semistable $E$ and $\operatorname{gr} E$ for the graded bundle associated to $E$; $\operatorname{gr} E$ depends only on $[E]$. The definition of $B_{n, e}^{k}(V)$ is extended to include semistable bundles by setting

$$
\widetilde{B}_{n, e}^{k}(V):=\left\{[E] \in U(n, e): h^{0}(C, V \otimes \operatorname{gr} E) \geq k\right\} .
$$

As outlined in [35, Sect. 1], the construction of $B_{n, e}^{k}$ in [13, Sect. 2] is easily generalised to $B_{n, e}^{k}(V)$, substituting a vector bundle $V$ for $\mathcal{O}_{C}$ in the appropriate places. (In Sect. 2 we will give a slightly more general version of this construction.) In particular, $B_{n, e}^{k}(V)$ is a determinantal locus. Thus, if $h^{0}(C, V \otimes E)=k$, then the expected dimension of $B_{n, e}^{k}(V)$ at $E$ is given by the twisted Brill-Noether number

$$
\begin{aligned}
\rho_{n, e}^{k}(V) & :=\operatorname{dim} U^{s}(n, e)-k(k-\chi(C, V \otimes E)) \\
& =n^{2}(g-1)+1-k(k-r e-n d+r n(g-1)) .
\end{aligned}
$$

Provided that this number is less than $\operatorname{dim} U^{s}(n, e)$, every irreducible component of $B_{n, e}^{k}(V)$ has dimension at least equal to $\rho_{n, e}^{k}(V)$. If $\rho_{n, e}^{k}(V) \geq \operatorname{dim} U^{s}(n, e)$ then $B_{n, e}^{k}(V)=U^{s}(n, e)$.

In the case $k=1$ with $V$ of integral slope $h=d / r$, we have $\rho_{1, g-1-h}^{1}(V)=g-1$ and $B_{1, g-1-h}^{1}(V)$ is expected to be a divisor $\Theta_{V}$ in the Picard variety $\operatorname{Pic}^{g-1-h}(C)$. When $\Theta_{V}$ is a divisor, it is called a generalised theta divisor. These have been much studied; see [2] for an overview. See also [8] for results on the singular loci of $\Theta_{V}$. It can also happen for special $V$ that $B_{1, g-1-h}^{1}(V)$ fails to be a divisor; see [28, 29,31] for some examples. If $V$ does not have integral slope, then the theta divisor of $V$, if it exists, belongs to $U^{s}(n, e)$ for some $n \geq 2$. See [30] for a survey of results on this type of generalised theta divisor.

Note also the connection with varieties of subbundles of a vector bundle $V$. If we denote by $M_{n, e}(V)$ the variety of stable subbundles of $V$ of rank $n$ and degree $e$, there is a natural morphism $M_{n, e}(V) \rightarrow B_{n,-e}^{1}(V)$ given by $E \mapsto E^{*}$. In particular, when $n=1$ and $e$ is maximal, this is a question of maximal line subbundles. In the case $r=2$, these have 
been studied for a long time, dating back to [19]; for more recent work and all $r$, see [27]. For $n>1$, see [16, 20, 32, Theorem 0.3] and [7].

When $n=1$, it turns out that the basic results of classical Brill-Noether theory generalise, at least when $V$ is a general stable bundle; for details, see Theorem 2.1. This study was initiated in [14]. Our purpose in this article is to study the case $n>1$.

In Sect. 2, we give more details on some of the background material mentioned in the introduction. In Sect. 3, we construct the twisted Brill-Noether locus $B^{k}(\mathcal{V}, \mathcal{E})$ associated to a pair of families of bundles over $C$, with $B_{n, e}^{k}(V)$ as a special case. After listing some elementary properties, we develop some more tools. We construct parameter spaces for certain "twisted coherent systems", generalising the loci $G_{d}^{r}(C)$ in [1] and the moduli spaces of $\alpha$-stable coherent systems, although we do not discuss stability or moduli.

In Sect. 4 we give two applications of the machinery set up in Sect. 3. In Theorem 4.1, we generalise Theorem 2.1(5) to families of vector bundles which are general in the sense of [35]. We also find that, for a certain range of values of $k$, the Brill-Noether locus $B_{r, d}^{k}$ possesses a uniquely determined irreducible component $\left(B_{r, d}^{k}\right)_{\mathrm{PTI}}$ (Theorem 4.3); this is interesting because very little is known in general about irreducibility of $B_{r, d}^{k}$ for $k \geq 2$ and $r>1$.

In Sect. 5, we turn to twisted Brill-Noether loci $B_{n, e}^{k}(V)$ for $n>1$ and $k \geq 2$, which to our knowledge remain relatively little studied. We will answer some of the basic questions on nonemptiness and smoothness in this case. Our first result is:

Theorem 1.1 Let $C$ be a smooth curve of genus $g \geq 2$ and $V$ any vector bundle of rank $r$ and degree $d$ over $C$. Let $e_{0}$ and $k_{0}$ be integers satisfying $\rho_{1, e_{0}}^{k_{0}}(V) \geq 1$. Then for all $n \geq 2$, for all $e \geq n e_{0}+1$ (resp., $e \geq n e_{0}$ ) and for $1 \leq k \leq n k_{0}$, the twisted Brill-Noether locus $B_{n, e}^{k}(V)$ (resp., $\tilde{B}_{n, e}^{k}(V)$ ) is nonempty.

This directly generalises both the main result and the construction of [24].

We are also interested in generically smooth components of the loci $B_{n, e}^{k}(V)$. Our approach turns out to require the existence of certain bundles with well-behaved rank-1 twisted Brill-Noether loci and which are generically generated. In Sect. 6 we construct such bundles for some values of $r, g$ and $d$. We then prove in Sect. 7 our main result:

Theorem 1.2 Let $C$ be a general curve of genus $g \geq 2$ and $r, l$, $m$ integers with $l:=\left\lfloor\frac{g}{r}\right\rfloor$ and $0 \leq m \leq l-1$. If $m=0$, suppose that $g \not \equiv 0 \bmod r$. Write $k_{0}=l-m$ and let $d, e_{0}$ be integers with $d+r e_{0}=r(g-2)+k_{0}$. Suppose that e and $k$ are integers satisfying

$$
n e_{0}+1 \leq e \leq n\left(e_{0}+1\right) \text { and } r e+n d-r n(g-1) \leq k \leq n k_{0} .
$$

Then, for general $V \in U^{s}(r, d)$, the twisted Brill-Noether locus $B_{n, e}^{k}(V)$ has a component $B_{n, e}^{k}(V)_{0}$ which is generically smooth and of the expected dimension.

Recall here that for $V \in U^{s}(r, d)$ and $E \in U^{s}(n, e)$, the Euler characteristic $\chi(V \otimes E)=r e+n d-r n(g-1)$ and moreover, if $k \leq \chi(V \otimes E)$, then $B_{n, e}^{k}(V)=U^{s}(n, e)$. The hypotheses of Theorem 1.2 may look rather restrictive. In fact, (1.2) is analogous to the conditions of $[24,33]$ for the case $r=1$. However, the restriction on $d+r e_{0}$ is strong and can probably be relaxed. 
To prove that $B_{n, e}^{k}(V)$ is generically smooth and of the expected dimension at a point $E$, we have to show that the generalised trace map

$$
H^{0}(C, V \otimes E) \otimes H^{0}\left(C, K_{C} \otimes E^{*} \otimes V^{*}\right) \rightarrow H^{0}\left(C, K_{C} \otimes \text { End } E\right)
$$

is injective (details in Sect. 3). For this type of question, Teixidor i Bigas's generalisation of limit linear series to vector bundles of higher rank has been applied in many situations; for example [10,33-35]. Although we do not use limit linear series directly, several of our proofs rely on the main result of [35].

In Sect. 8, we consider the tangent cones of $B_{n, e}^{k}(V)$, which can be studied using the same techniques as in $[1,9]$. Using Theorem 1.2, we describe the tangent cones as determinantal varieties and compute their degrees. We also give a geometric description of the tangent cones for large values of $h^{0}(C, V \otimes E)$, generalising [1, VI, Theorem 1.6 (i)] on secant varieties of canonical curves.

Finally, in Sect. 9, we describe some twisted Brill-Noether loci which are nonempty but have negative expected dimension. These are closely connected with varieties of maximal subbundles, and we exploit results of [27] in discussing them. This gives another motivation for studying twisted Brill-Noether loci: as these examples arise for general $C$ and $V$ (with prescribed numerical properties), twisted Brill-Noether loci give a way of systematically obtaining determinantal varieties of larger than expected dimension. This line of research will be further pursued in the future.

Notation We work over an algebraically closed field $\mathbb{K}$ of characteristic zero. We denote a locally free sheaf and the corresponding vector bundle by the same letter. If $F$ is an $\mathcal{O}_{C^{-}}$ module, we abbreviate $H^{i}(C, F), h^{i}(C, F)$ and $\chi(C, F)$, respectively, to $H^{i}(F), h^{i}(F)$ and $\chi(F)$. If $D$ is a divisor on $C$, we denote $F \otimes \mathcal{O}_{C}(D)$ by $F(D)$. The fibre of a bundle $V$ at $p \in C$ will be denoted $\left.V\right|_{p}$. If $\mathcal{V} \rightarrow S \times C$ is a family of bundles parametrised by $S$, we denote the restriction $\left.\mathcal{V}\right|_{\{s\} \times C}$ by $\mathcal{V}_{s}$. We suppose throughout that $g \geq 2$ and $k \geq 1$.

\section{Background}

In this section, we expand on the background to our paper already referred to in the introduction. The fundamental results on $W_{d}^{r}(C)$ are as follows (see [1, Chapter V]):

(i) Existence theorem: For any curve, $W_{d}^{r}(C)$ is nonempty if $\rho(g, d, r) \geq 0$.

(ii) Connectedness theorem: For any curve, $W_{d}^{r}(C)$ is connected if $\rho(g, d, r) \geq 1$.

(iii) Dimension theorem: For a general curve, $W_{d}^{r}(C)=\emptyset$ if $\rho(g, d, r)<0$; if $0 \leq \rho(g, d, r) \leq g$, then $W_{d}^{r}(C)$ has pure dimension $\rho(g, d, r)$.

(iv) Smoothness theorem: For a general curve and $\rho(g, d, r)<g, \operatorname{Sing}\left(W_{d}^{r}(C)\right)=W_{d}^{r+1}(C)$.

(v) For a general curve, $W_{d}^{r}(C)$ is irreducible if $\rho(g, d, r) \geq 1$.

Note that if $\rho(g, d, r) \geq g$, then $W_{d}^{r}(C)=\operatorname{Pic}^{d}(C)$.

Many of the basic questions on nonemptiness of $B_{n, e}^{k}$ were answered in [24, 33], and more detailed results have been obtained in several cases. However, analogues of statements (i)-(v) may be false in higher rank. See [13] for an overview of the theory and a survey of results and techniques. For the links between Brill-Noether theory and the moduli of coherent systems, see [4,5]. See also [26] for a survey of results and open problems on coherent systems; note however that there are many more recent results in this area. 
When $B_{n, e}^{k}(V)$ has the expected dimension, one has

$$
B_{n, e}^{k+1}(V) \subseteq \operatorname{Sing}\left(B_{n, e}^{k}(V)\right) .
$$

This containment may, however, be strict. See [9] for a detailed discussion of singular points $E \in \Theta_{V}$ satisfying mult ${ }_{E}\left(\Theta_{V}\right)>h^{0}(V \otimes E)$.

As already remarked, for $n=1$, analogues of several of the fundamental results for $W_{d}^{r}(C)$ are also valid for sufficiently general bundles of higher rank (by "general", we mean stable and general in the moduli space):

Theorem 2.1 Let $C$ be any curve of genus $g \geq 2$. Let $V$ be a vector bundle of rank $r$ and degree $d$. Let $k \geq 1$ and e be integers.

(1) If $\rho_{1, e}^{k}(V) \geq 0$, then $B_{1, e}^{k}(V)$ is nonempty.

(2) If $\rho_{1, e}^{k}(V) \geq 1$, then $B_{1, e}^{k}(V)$ is connected.

(3) Suppose $C$ is a general curve and $V$ a general bundle. If $\rho_{1, e}^{k}(V)<0$, then $B_{1, e}^{k}(V)$ is empty. If $0 \leq \rho_{1, e}^{k}(V) \leq g$, then $B_{1, e}^{k}(V)$ has pure dimension $\rho_{1, e}^{k}(V)$.

(4) Suppose $C$ is a general curve and $V$ a general bundle. If $\rho_{1, e}^{k}(V)<g$, then $\operatorname{Sing}\left(B_{1, e}^{k}(V)\right)=B_{1, e}^{k+1}(V)$.

(5) Suppose C is a Petri curve and V a general bundle. If $\rho_{1, e}^{k}(V) \geq 1$, then $B_{1, e}^{k}(V)$ is irreducible.

Proof Statement (1) was proven in [12] for general $V$, and for all $V$ in [22, (2.6)]. Part (2) is $[22,(2.7)]$. Parts (3) and (4) follow from [35]. Lastly, (5) is [14, Théorème 1.2].

The infinitesimal study of the Brill-Noether loci is the key to the proofs of (3) and (4). We do not include this here because we will describe it in detail for $B_{n, e}^{k}(V)$ (and indeed for families of bundles) in the next section.

\section{Preliminaries on twisted Brill-Noether loci}

Although generalised Brill-Noether loci are by now very familiar objects, there are fewer sources focusing primarily on twisted Brill-Noether loci. We will therefore give a detailed introduction to the subject with emphasis on functorial aspects.

\subsection{The twisted Brill-Noether locus of a pair of families}

Let $\mathcal{V} \rightarrow S \times C$ be a family of bundles of rank $r$ and degree $d$, and $\mathcal{E} \rightarrow T \times C$ a family of bundles of rank $n$ and degree $e$. Set-theoretically, we define

$$
B^{k}(\mathcal{V}, \mathcal{E}):=\left\{(s, t) \in S \times T: h^{0}\left(\mathcal{V}_{s} \otimes \mathcal{E}_{t}\right) \geq k\right\}
$$

Scheme-theoretically, this is a determinantal locus, as we will now show using a standard construction. Let $D$ be an effective divisor of large degree on $C$ satisfying $h^{1}\left(\mathcal{V}_{s} \otimes \mathcal{E}_{t}(D)\right)=0$ for all $(s, t) \in S \times T$. We have a diagram of projections 


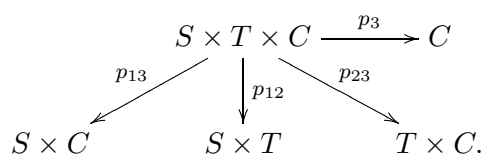

Then over $S \times T \times C$, we have the short exact sequence

$$
0 \rightarrow p_{13}^{*} \mathcal{V} \otimes p_{23}^{*} \mathcal{E} \rightarrow p_{13}^{*} \mathcal{V} \otimes p_{23}^{*} \mathcal{E} \otimes p_{3}^{*} \mathcal{O}_{C}(D) \rightarrow \frac{p_{13}^{*} \mathcal{V} \otimes p_{23}^{*} \mathcal{E} \otimes p_{3}^{*} \mathcal{O}_{C}(D)}{p_{13}^{*} \mathcal{V} \otimes p_{23}^{*} \mathcal{E}} \rightarrow 0
$$

Pushing down to $S \times T$, we obtain a complex $\gamma: K^{0} \rightarrow K^{1}$ of locally free sheaves satisfying

$$
\operatorname{Ker}\left(\gamma_{(s, t)}\right) \cong H^{0}\left(\mathcal{V}_{s} \otimes \mathcal{E}_{t}\right) \text { and } \operatorname{Coker}\left(\gamma_{(s, t)}\right) \cong H^{1}\left(\mathcal{V}_{s} \otimes \mathcal{E}_{t}\right)
$$

for each $(s, t) \in S \times T$. Then $B^{k}(\mathcal{V}, \mathcal{E})$ is the locus defined by the $\left(\operatorname{rk} K^{0}-k\right) \times\left(\operatorname{rk} K^{0}-k\right)$ minors of $\gamma$. In particular (see [1, Chapter 2]), the locus $B^{k}(\mathcal{V}, \mathcal{E})$ has a natural scheme structure and every component of it has dimension at least

$$
\operatorname{dim} S+\operatorname{dim} T-k(k-r e-n d+r n(g-1)) .
$$

From the determinantal description it also follows that $B^{k+1}(\mathcal{V}, \mathcal{E}) \subseteq \operatorname{Sing}\left(B^{k}(\mathcal{V}, \mathcal{E})\right)$, and moreover that the loci $B^{k}(\mathcal{V}, \mathcal{E})$ define a rank stratification on $S \times T$. We will return to this aspect in Sect. 3.5.

Remark 3.1 This construction is symmetric in $\mathcal{V}$ and $\mathcal{E}$. It is functorial in the sense that if $\phi: S^{\prime} \rightarrow S$ and $\psi: T^{\prime} \rightarrow T$ are morphisms, then

$$
B^{k}\left(\left(\phi \times \mathrm{Id}_{C}\right)^{*} \mathcal{V},\left(\psi \times \mathrm{Id}_{C}\right)^{*} \mathcal{E}\right)
$$

is defined by the $\left(\operatorname{rk} K^{0}-k\right) \times\left(\operatorname{rk} K^{0}-k\right)$-minors of $(\phi \times \psi)^{*} \gamma$.

Definition 3.2 Let $V$ be a vector bundle of rank $r$ and degree $d$, considered as a family over Spec $\mathbb{K} \times C$. By [25, Proposition 2.4], there exists an étale cover $\widetilde{U^{s}(n, e)} \rightarrow U^{s}(n, e)$ (which can be taken to be the identity if $\operatorname{gcd}(n, e)=1$ ) which carries a Poincaré family $\mathcal{E} \rightarrow \widehat{U^{s}(n, e)} \times C$. Then the twisted Brill-Noether locus $B_{n, e}^{k}(V)$ is defined as the image of the moduli map $B^{k}(V, \mathcal{E}) \rightarrow U^{s}(n, e)$. Writing $\chi=r e+n d-r n(g-1)$, the expected dimension of $B_{n, e}^{k}(V)$ is the Brill-Noether number

$$
\rho_{n, e}^{k}(V)=\rho_{n, e, r, d}^{k}:=\operatorname{dim} U^{s}(n, e)-k(k-\chi) .
$$

The following is straightforward to check:

Proposition 3.3 Let $V$ be any bundle of rank $r$ and degree $d$ over $C$.

(1) $B_{n, e}^{k}(V)$ is a proper sublocus of $U^{s}(n, e)$ only if $k>\chi$.

(2) If $V$ is stable, then $B_{n, e}^{k}(V)$ is nonempty only if re $+n d>0$ or $(n, e)=(r,-d)$. In the latter case, $B_{r,-d}^{1}(V) \stackrel{n, e}{=}\left\{V^{*}\right\}$ and $B_{r,-d}^{k}$ is empty for $k \geq 2$.

(3) If $V$ is semistable, then $B_{n, e}^{k}(V)$ is nonempty only if re $+n d>0$ or $r e+n d=0$ and $r \geq n$.

(4) For any line bundle L of degree $\ell$, there is a canonical isomorphism 


$$
B_{n, e}^{k}(V) \stackrel{\sim}{\longrightarrow} B_{n, e-n \ell}^{k}(V \otimes L)
$$

given by $E \mapsto L^{-1} \otimes E$.

(5) Via Serre duality, the association $E \mapsto E^{*}$ gives an isomorphism

$$
B_{n, e}^{k}(V) \stackrel{\sim}{\longrightarrow} B_{n,-e}^{k-\chi}\left(K_{C} \otimes V^{*}\right) .
$$

Remark 3.4 If $r=1$, there are many examples of Brill-Noether loci which are empty although $\rho_{n, e, r, d}^{k} \geq 0$ (see, for example, [6,23]). It seems to be much harder to find examples for $r>1$, but Proposition 3.3 does provide some. Suppose that $r e+n d=0$ and $r<n$; then $B_{n, e}^{1}(V)=\emptyset$ by Proposition 3.3(2). On the other hand, in this case,

$$
\rho_{n, e, r, d}^{1}=n^{2}(g-1)+1-(1+r n(g-1))=n(n-r)(g-1)>0 .
$$

Furthermore, it was noted in [6] that, for $r \geq 2$, the locus $B_{r, 0}^{k}$ is empty, but $\widetilde{B}_{r, 0}^{k} \neq \emptyset$ for $k \leq r$. We can generalise this example to twisted Brill-Noether loci. Suppose $V \stackrel{r, 0}{\in} U^{s}(r, d)$ is a stable bundle, $n>r$ and $r e+n d=0$. Then, by Proposition $3.3(2), B_{n, e}^{k}(V)=\emptyset$ for all $k$. However, taking $E=V^{*} \oplus F$, where $F$ is semistable and $\mu(F)=\mu(E)$, we see that $[E] \in \widetilde{B}_{n, e}^{1}(V)$.

\subsection{The tangent spaces of $B^{k}(\mathcal{V}, \mathcal{E})$}

We now recall some standard facts on deformations of bundles and sections. Suppose $W$ is a vector bundle with $h^{0}(W) \geq 1$. Let $v \in H^{1}$ (End $W$ ) be a first-order infinitesimal deformation of $W$. By the argument in [13, Sect. 2], a section $s \in H^{0}(W)$ is preserved by $v$ if and only if $s \cup v=0$ in $H^{1}(W)$. Thus the space of deformations preserving all sections of $W$ is exactly

$$
\operatorname{Ker}\left(\cup: H^{1}(\text { End } W) \rightarrow \operatorname{Hom}\left(H^{0}(W), H^{1}(W)\right)\right) .
$$

We are interested in the case where $W$ is of the form $V \otimes E$ and $v$ is the class of a product of deformations $b \in H^{1}$ (End $V$ ) and $h \in H^{1}($ End $E)$ of $V$ and $E$, respectively. By for example inspecting Čech cocycles, we see that $v=c(b, h)$, where

$$
c(b, h):=b \otimes \operatorname{Id}_{E}+\operatorname{Id}_{V} \otimes h \in H^{1}(\operatorname{End}(V \otimes E)) .
$$

More generally, let us consider again the families $\mathcal{V} \rightarrow S \times C$ and $\mathcal{E} \rightarrow T \times C$. Suppose $(s, t) \in S \times T$ is such that $h^{0}\left(\mathcal{V}_{s} \otimes \mathcal{E}_{t}\right)=k$. We have a composed map

$$
\begin{aligned}
T_{s} S \oplus T_{t} T \stackrel{\kappa}{\rightarrow} H^{1}\left(\text { End } \mathcal{V}_{s}\right) \oplus H^{1}\left(\text { End } \mathcal{E}_{t}\right) \stackrel{c}{\rightarrow} H^{1}\left(\text { End }\left(\mathcal{V}_{s} \otimes \mathcal{E}_{t}\right)\right) \\
\stackrel{\cup}{\rightarrow} \operatorname{Hom}\left(H^{0}\left(\mathcal{V}_{s} \otimes \mathcal{E}_{t}\right), H^{1}\left(\mathcal{V}_{s} \otimes \mathcal{E}_{t}\right)\right)
\end{aligned}
$$

where $\kappa$ is the Kodaira-Spencer map.

Proposition 3.5 Suppose that $h^{0}\left(\mathcal{V}_{s} \otimes \mathcal{E}_{t}\right)=k$. 
(1) The Zariski tangent space to $B^{k}(\mathcal{V}, \mathcal{E})$ at $(s, t)$ is given by

$$
T_{(s, t)} B^{k}(\mathcal{V}, \mathcal{E})=\operatorname{Ker}(\cup \circ \circ \circ \kappa)
$$

(2) In particular, suppose that $S \times T$ is smooth at $(s, t)$. Then $B^{k}(\mathcal{V}, \mathcal{E})$ is smooth and of expected dimension (3.1) at $(s, t)$ if and only if $\cup \circ \mathrm{\circ} \kappa$ is surjective.

Proof Since $T_{(s, t)} B^{k}(\mathcal{V}, \mathcal{E})$ consists of those deformations preserving all sections of $\mathcal{V}_{s} \otimes \mathcal{E}_{t}$, we obtain (1). By (3.1) and (1), we see that $B^{k}(\mathcal{V}, \mathcal{E})$ is smooth of the expected dimension at $(s, t)$ if and only if

$$
\operatorname{dim} S+\operatorname{dim} T-\operatorname{dim} \operatorname{Ker}(\cup \circ c \circ \kappa)=k(k-r e-n d+r n(g-1)) .
$$

By (3.3), this is equivalent to the surjectivity of $\cup \circ \circ \kappa$.

\subsection{The Petri trace map}

For any vector bundle $W$, it is well known that, via Serre duality, $\cup: H^{1}(\operatorname{End}(W)) \rightarrow \operatorname{Hom}\left(H^{0}(W), H^{1}(W)\right)$ is dual to the Petri multiplication map

$$
\mu: H^{0}(W) \otimes H^{0}\left(K_{C} \otimes W^{*}\right) \rightarrow H^{0}\left(K_{C} \otimes \text { End } W\right) .
$$

Let us use this map to reformulate (3.4).

Firstly, some notation: for bundles $V$ and $E$, there is a vector bundle map

$$
c:(\text { End } V) \oplus(\text { End } E) \rightarrow \text { End }(V \otimes E)
$$

inducing the cohomology map (3.2) considered above. We write $c_{V}$ and $c_{E}$ for the restrictions to the first and second factors, respectively. Recall also that for any bundle $W$, the transpose gives a canonical identification of End $W$ and End $W^{*}$, which we will use freely.

Fix a vector bundle $V$. If we identify End $V$ with (End $V)^{*}$ by the trace pairing, a diagram chase shows that the trace map $\operatorname{tr}:$ End $V \rightarrow \mathcal{O}_{C}$ is dual to the map $\mathcal{O}_{C} \rightarrow$ End $V$ given by $\lambda \mapsto \lambda \cdot \operatorname{Id}_{V}$.

Thus for any bundle $E$, tensoring tr : End $V \rightarrow \mathcal{O}_{C}$ by End $E$, we obtain a linear map

$$
\text { End }(V \otimes E) \cong \text { End } V \otimes \text { End } E \rightarrow \text { End } E
$$

which is dual to $c_{E}$, and an induced map

$$
\operatorname{tr}_{E}: H^{0}\left(K_{C} \otimes \operatorname{End}(V \otimes E)\right) \rightarrow H^{0}\left(K_{C} \otimes \operatorname{End} E\right) .
$$

By Serre duality and the above discussion, $\operatorname{tr}_{E}$ is dual to

$$
c_{E}: H^{1}(\text { End } E) \rightarrow H^{1}(\text { End }(V \otimes E)) .
$$

Then, by linear algebra, $c: H^{1}($ End $V) \oplus H^{1}($ End $E) \rightarrow H^{1}($ End $(V \otimes E))$ is dual to

$$
\left(\operatorname{tr}_{V}, \operatorname{tr}_{E}\right): H^{0}\left(K_{C} \otimes \text { End }(V \otimes E)\right) \rightarrow H^{0}\left(K_{C} \otimes \text { End } V\right) \oplus H^{0}\left(K_{C} \otimes \text { End } E\right) .
$$

We can now formulate a dual version of Proposition 3.5. 
Proposition 3.6 Suppose $h^{0}\left(\mathcal{V}_{s} \otimes \mathcal{E}_{t}\right)=k$. The Zariski tangent space $T_{(s, t)} B^{k}(\mathcal{V}, \mathcal{E})$ is the annihilator of the image of

$$
{ }^{t} \kappa \circ\left(\operatorname{tr}_{\mathcal{V}_{s}}, \operatorname{tr}_{\mathcal{E}_{t}}\right) \circ \mu: H^{0}\left(\mathcal{V}_{s} \otimes \mathcal{E}_{t}\right) \otimes H^{0}\left(K_{C} \otimes \mathcal{E}_{t}^{*} \otimes \mathcal{V}_{s}^{*}\right) \rightarrow T_{s}^{*} S \oplus T_{t}^{*} T .
$$

In particular, if $S \times T$ is smooth at $(s, t)$, then $B^{k}(\mathcal{V}, \mathcal{E})$ is smooth and of the expected dimension at $(s, t)$ if and only if $\left.{ }^{t} \kappa \circ\left(\operatorname{tr}_{\mathcal{V}_{s}}, \operatorname{tr}_{\mathcal{E}_{t}}\right)\right) \circ \mu$ is injective.

The most important corollary of this proposition is:

Corollary 3.7 Let $V$ be a bundle of rank $r$ and degree $d$. Suppose $E \in U^{s}(n, e)$ satisfies $h^{0}(V \otimes E)=k$. The twisted Brill-Noether locus $B_{n, e}^{k}(V)$ is smooth and of the expected dimension at $E$ if and only if

$$
\begin{aligned}
\operatorname{tr}_{E} \circ \mu: H^{0}(V \otimes E) \otimes H^{0}\left(K_{C} \otimes E^{*} \otimes V^{*}\right) & \rightarrow H^{0}\left(K_{C} \otimes \text { End }(V \otimes E)\right) \\
& \rightarrow H^{0}\left(K_{C} \otimes \text { End } E\right)
\end{aligned}
$$

is injective.

Proof This follows from Proposition 3.6 applied to the family $\mathcal{V}$ consisting of the single bundle $V$ and a local universal family $\mathcal{E}$ for $E$, together with the fact that the Kodaira-Spencer map for the family $\mathcal{E}$ at $E$ is an isomorphism.

It will be convenient to make the following definition (see also [15, Definition 2.1]).

Definition 3.8 For fixed $V$, write $\mu_{E}$ for the composed map $\operatorname{tr}_{E} \circ \mu$ in (3.5). We say that $V$ is Petri E-trace injective if $\mu_{E}$ is injective. If the trace map

$$
\mu_{\mathcal{O}_{C}}: H^{0}(V) \otimes H^{0}\left(K_{C} \otimes V^{*}\right) \rightarrow H^{0}\left(K_{C}\right)
$$

is injective, we say that $V$ is Petri trace injective.

Next, as it will be central to several proofs, let us state [35, Theorem 1.1] precisely.

Theorem 3.9 Let $C$ be a general curve and $V$ a general vector bundle over $C$. Then for any degree $e$ and $L \in \operatorname{Pic}^{e}(C)$, the Petri trace map

$$
\mu_{L}: H^{0}(V \otimes L) \otimes H^{0}\left(K_{C} \otimes L^{-1} \otimes V^{*}\right) \rightarrow H^{0}\left(K_{C}\right)
$$

is injective.

This motivates another definition.

Definition 3.10 A vector bundle $V$ is Petri general if $V$ is Petri $L$-trace injective for all $L \in \operatorname{Pic}(C)$.

Remark 3.11 A curve $C$ is Petri in the usual sense if and only if $\mathcal{O}_{C}$ is a Petri general vector bundle. It is well known that the general curve $C$ is a Petri curve. 


\subsection{A partial desingularisation of $B^{k}(\mathcal{V}, \mathcal{E})$}

Here we generalise the construction $G_{d}^{r}(C)$ of [1, IV.4] to twisted Brill-Noether loci.

For families $\mathcal{V}$ and $\mathcal{E}$, let us fix the effective divisor $D$ in Sect. 3.1 and recall the complex $\gamma: K^{0} \rightarrow K^{1}$. As $K^{0}$ is locally free, we have a Grassmannian bundle $\pi: \operatorname{Gr}\left(k, K^{0}\right) \rightarrow S \times T$. We define

$$
G^{k}(\mathcal{V}, \mathcal{E}):=\left\{\Lambda \in \operatorname{Gr}\left(k, K^{0}\right):\left.\gamma\right|_{\Lambda}=0\right\}
$$

This is a parameter space for triples $(V, E, \Lambda)$ where $\Lambda$ is a $k$-dimensional subspace of $H^{0}(V \otimes E)$. It seems natural to call such a triple a "twisted coherent system", but we do not pursue questions of moduli or stability here. When the family $\mathcal{V}$ consists of a single vector bundle $V$, we write also $G^{k}(V, \mathcal{E})$.

Clearly, $\pi\left(G^{k}(\mathcal{V}, \mathcal{E})\right)=B^{k}(\mathcal{V}, \mathcal{E})$ and $\pi^{-1}(s, t)=\operatorname{Gr}\left(k, H^{0}\left(\mathcal{V}_{s} \otimes \mathcal{E}_{t}\right)\right)$. Let us describe the Zariski tangent spaces of $G^{k}(\mathcal{V}, \mathcal{E})$ at $(s, t)$.

\section{Proposition 3.12}

(1) Suppose $h^{0}\left(\mathcal{V}_{s} \otimes \mathcal{E}_{t}\right) \geq k$. There is an exact sequence

$$
\begin{aligned}
0 \rightarrow \operatorname{Hom}\left(\Lambda, H^{0}\left(\mathcal{V}_{s} \otimes \mathcal{E}_{t}\right) / \Lambda\right) & \rightarrow T_{(\Lambda, s, t)} G^{k}(\mathcal{V}, \mathcal{E}) \stackrel{d \pi}{\longrightarrow} T_{s} S \oplus T_{t} T \\
& \rightarrow \operatorname{Hom}\left(\Lambda, H^{1}\left(\mathcal{V}_{s} \otimes \mathcal{E}_{t}\right)\right)
\end{aligned}
$$

where the last map is defined by $\cup \circ \mathrm{\circ} \circ \mathrm{K}$ as in (3.3), followed by restriction to $\Lambda$. Moreover, $\operatorname{Im}(d \pi)=\operatorname{Im}\left({ }^{t} \kappa \circ\left(\operatorname{tr}_{\mathcal{V}_{s}}, \operatorname{tr}_{\mathcal{E}_{t}}\right) \circ \mu\right)^{\perp}$.

(2) The locus $G^{k}(\mathcal{V}, \mathcal{E})$ is smooth and of dimension (3.1) at $(s, t, \Lambda)$ if and only if the restricted map

${ }^{t} \kappa \circ\left(\operatorname{tr}_{\mathcal{V}_{s}}, \operatorname{tr}_{\mathcal{E}_{t}}\right) \circ \mu: \Lambda \otimes H^{0}\left(K_{C} \otimes \mathcal{E}_{t}^{*} \otimes \mathcal{V}_{s}^{*}\right) \rightarrow H^{0}\left(K_{C} \otimes\right.$ End $\left.\mathcal{V}_{s}\right) \oplus H^{0}\left(K_{C} \otimes\right.$ End $\left.\mathcal{E}_{t}\right)$

is injective.

(3) In particular, if ${ }^{t} \kappa \circ\left(\operatorname{tr}_{\mathcal{V}_{s}}, \operatorname{tr}_{\mathcal{E}_{t}}\right) \circ \mu$ is injective and $h^{0}\left(\mathcal{V}_{s} \otimes \mathcal{E}_{t}\right)>k$, then $G^{k}(\mathcal{V}, \mathcal{E})$ is a desingularisation of $B^{k}(\mathcal{V}, \mathcal{E})$ in a neighbourhood of $(s, t)$.

Proof Statements (1) and (2) are proven in the same way as [1, Proposition IV.4.1 (ii)(iii), p. 187], and clearly (3) follows from (2).

\subsection{A sufficient condition for the existence of good components}

Note that

$$
S \times T \supset B^{1}(\mathcal{V}, \mathcal{E}) \supset B^{2}(\mathcal{V}, \mathcal{E}) \supset \cdots \supset B^{k}(\mathcal{V}, \mathcal{E})
$$

is a stratification of $B^{k}(\mathcal{V}, \mathcal{E})$ by closed subsets. The following proposition makes use of this stratification.

Proposition 3.13 Suppose $S \times T$ is smooth at $(s, t)$ and for some $k^{\prime} \geq \chi$ there exists $(s, t) \in B^{k^{\prime}}(\mathcal{V}, \mathcal{E})$ such that $h^{0}\left(\mathcal{V}_{s} \otimes \mathcal{E}_{t}\right)=k^{\prime}$ and ${ }^{t} \kappa \circ\left(\operatorname{tr}_{\mathcal{V}_{s}}, \operatorname{tr}_{\mathcal{E}_{t}}\right) \circ \mu$ is injective. Then, for 
$\chi \leq k \leq k^{\prime}$, the locus $B^{k}(\mathcal{V}, \mathcal{E})$ contains a component which is generically smooth and of the expected dimension.

Proof We prove this by descending induction on $k$. For $k=k^{\prime}$, the result follows immediately from Proposition 3.6. Now suppose $\chi \leq k<k^{\prime}$ and that the proposition holds for $B^{k+1}(\mathcal{V}, \mathcal{E})$. Then, there exists $(V, E) \in B^{k+1}(\mathcal{V}, \mathcal{E})$ with $h^{0}(V \otimes E)=k+1$ and ${ }^{t} \kappa \circ\left(\operatorname{tr}_{V}, \operatorname{tr}_{E}\right) \circ \mu$ injective. Now let $\Lambda$ be any $k$-dimensional linear subspace of $H^{0}(V \otimes E)$. Then, by Proposition $3.12, G^{k}(\mathcal{V}, \mathcal{E})$ is smooth of the expected dimension at $(V, E, \Lambda)$. Since $k \geq \chi$, it follows from (3.1) that every component of $G^{k}(\mathcal{V}, \mathcal{E})$ has dimension greater than the dimension of $\pi^{-1}\left(B^{k+1}(\mathcal{V}, \mathcal{E})\right)$ at $(V, E)$. Hence, there exists a point $\left(V_{1}, E_{1}, \Lambda_{1}\right)$ of $G^{k}(\mathcal{V}, \mathcal{E})$ in the neighbourhood of $(V, E, \Lambda)$ with $h^{0}\left(V_{1} \otimes E_{1}\right)=k$ and ${ }^{t} \kappa \circ\left(\operatorname{tr}_{V_{1}}, \operatorname{tr}_{E_{1}}\right) \circ \mu$ injective. Thus $B^{k}(\mathcal{V}, \mathcal{E})$ is smooth of the expected dimension at $\left(V_{1}, E_{1}\right)$.

This proposition illustrates a general principle that, from the existence of just one pair of bundles with good properties, one can obtain a detailed picture of the geometry of several of the strata. This will be used on a number of occasions later.

\section{Two irreducibility results}

Here we will give some applications of the machinery assembled in the previous section.

\subsection{Rank one twisted Brill-Noether loci}

If $C$ is a Petri curve, $B_{1, e}^{k}=W_{e}^{k-1}(C)$ is irreducible whenever $\rho_{1, e}^{k}=g-k(k-e+g-1) \geq 1$. Let $\mathcal{P} \rightarrow \operatorname{Pic}^{e}(C) \times C$ be a Poincaré bundle.

Theorem 4.1 Let $\mathcal{V} \rightarrow S \times C$ be a family of Petri general vector bundles of rank $r$ and degree d parametrised by a smooth irreducible base S. Assume that

$$
\rho_{1, e, r, d}^{k}=g-k(k-(d+r e)+r(g-1)) \geq 1 .
$$

Then $G^{k}(\mathcal{V}, \mathcal{P})$ is smooth and irreducible, and $B^{k}(\mathcal{V}, \mathcal{P}) \subseteq S \times \operatorname{Pic}^{e}(C)$ is an irreducible variety of dimension $\operatorname{dim} S+\rho_{1, e, r, d}^{k}$ which is singular precisely along $B^{k+1}(\mathcal{V}, \mathcal{P})$.

Proof The fibre of $B^{k}(\mathcal{V}, \mathcal{P})$ over each $s \in S$ is exactly $B_{1, e}^{k}\left(\mathcal{V}_{s}\right)$. Since $\rho_{1, e, r, d}^{k} \geq 1$, by Theorem 2.1(1) and (2) this fibre is nonempty and connected. As $S$ is irreducible, it follows that $B^{k}(\mathcal{V}, \mathcal{P})$ is connected. As the fibres of $G^{k}(\mathcal{V}, \mathcal{P}) \rightarrow B^{k}(\mathcal{V}, \mathcal{P})$ are Grassmannians, $G^{k}(\mathcal{V}, \mathcal{P})$ is also connected.

Furthermore, as $\mathcal{V}_{s}$ is Petri general for all $s$, by Proposition 3.12(2) in fact $G^{k}(\mathcal{V}, \mathcal{P})$ is smooth. Therefore $G^{k}(\mathcal{V}, \mathcal{P})$ is irreducible. As $B^{k}(\mathcal{V}, \mathcal{P})$ is the image of $G^{k}(\mathcal{V}, \mathcal{P})$ by a morphism, $B^{k}(\mathcal{V}, \mathcal{P})$ is also irreducible.

The last statement follows from Petri generality and Proposition 3.6.

Remark 4.2 Suppose that $C$ is a general curve. Since, by Theorem 3.9, a general bundle $V$ in $U^{s}(r, d)$ is Petri general, in particular $B_{1, e}^{k}(V)$ is irreducible for general $V$. Thus we recover [14, Théorème 1.2]. 


\subsection{Irreducibility of Petri trace injective loci}

Here we give an application to "nontwisted" Brill-Noether loci.

Theorem 4.3 Suppose $C$ is general and $g \geq \rho_{1,0, r, d}^{k}=g-k(k-d+r(g-1)) \geq 1$. Then there is a unique irreducible component $\left(B_{r, d}^{k}\right)_{\mathrm{PTI}}$ of the Brill-Noether locus $B_{r, d}^{k}$ containing the locus

$$
\left\{V \in B_{r, d}^{k}: \bar{\mu}: \Lambda \otimes H^{0}\left(K_{C} \otimes V^{*}\right) \rightarrow H^{0}\left(K_{C}\right) \text { is injective for some } \Lambda \in \operatorname{Gr}\left(k, H^{0}(V)\right)\right\}
$$

In particular, the locus of Petri trace injective bundles in $B_{r, d}^{k}$ is irreducible.

Proof By [25, Proposition 2.6], there exists a smooth irreducible variety $\mathcal{M}$ admitting a Poincaré family $\mathcal{V} \rightarrow \mathcal{M} \times C$ such that every stable bundle of rank $r$ and degree $d$ over $C$ is represented in $\mathcal{M}$. Throughout, we will write $\widetilde{V}$ for a point of $\mathcal{M}$ lying over $V \in U^{s}(r, d)$.

Set $e=0$ and let $\mathcal{P} \rightarrow \operatorname{Pic}^{0}(C) \times C$ be a Poincaré bundle. We consider the locus $G^{k}(\mathcal{V}, \mathcal{P}) \rightarrow \mathcal{M} \times \operatorname{Pic}^{0}(C)$ defined in Sect. 3.4. Note that the projection map

$$
p: G^{k}(\mathcal{V}, \mathcal{P}) \rightarrow \mathcal{M} \times \operatorname{Pic}^{0}(C) \rightarrow \mathcal{M}
$$

is surjective by Theorem 2.1(1). Define

$$
\mathcal{G}^{k}:=\left\{(\widetilde{V}, L, \Lambda) \in G^{k}(\mathcal{V}, \mathcal{P}): \mu_{L}: \Lambda \otimes H^{0}\left(K_{C} \otimes L^{-1} \otimes V^{*}\right) \rightarrow H^{0}\left(K_{C}\right) \text { is injective }\right\} .
$$

By Theorem 2.1(3), (4) and by hypothesis, this is nonempty. It is also open in $G^{k}(\mathcal{V}, \mathcal{P})$ and smooth of pure dimension

$$
\operatorname{dim} \mathcal{M}+\operatorname{dim} \operatorname{Pic}^{0}(C)-k(k-d+r(g-1))=\operatorname{dim} \mathcal{M}+\rho_{1,0, r, d}^{k}
$$

by Proposition 3.12(2).

Let $t: G^{k}(\mathcal{V}, \mathcal{P}) \rightarrow U^{s}(r, d)$ be the morphism given by $t(\widetilde{V}, L, \Lambda)=V \otimes L$. By definition, $t\left(\mathcal{G}^{k}\right)$ is locus (4.1). Thus it will suffice to show that $\mathcal{G}^{k}$ is irreducible.

Since the projection $p$ of (4.2) is surjective, there is at least one irreducible component of $\mathcal{G}^{k}$ which dominates $\mathcal{M}$. We claim first that this component is unique. Suppose that $X_{1}$ and $X_{2}$ were components of $\mathcal{G}^{k}$ such that $p\left(X_{1}\right)$ and $p\left(X_{2}\right)$ are both dense in $\mathcal{M}$. Let $\widetilde{V}$ be a general point of $p\left(X_{1}\right) \cap p\left(X_{2}\right)$. The fibre $p^{-1}(\widetilde{V})$ is identified with

$$
G^{k}(\widetilde{V}, \mathcal{P})=\left\{(L, \Lambda): \Lambda \in \operatorname{Gr}\left(k, H^{0}(\widetilde{V} \otimes L)\right)\right\},
$$

here viewing $\widetilde{V}$ as a singleton family. By Theorem 3.9, we may assume $V$ is Petri general. Since $\rho_{1,0, r, d}^{k} \geq 1$ by hypothesis, it follows from Theorem 4.1 that $G^{k}(\widetilde{V}, \mathcal{P})$ is irreducible. Since $X_{1} \cap G^{k}(\widetilde{V}, \mathcal{P})$ and $X_{2} \cap G^{k}(\widetilde{V}, \mathcal{P})$ are both components of $\mathcal{G}^{k} \cap G^{k}(\widetilde{V}, \mathcal{P})$, it follows that $X_{1} \cap G^{k}(\widetilde{V}, \mathcal{P})=X_{2} \cap G^{k}(\widetilde{V}, \mathcal{P})$ and, in particular, $X_{1} \cap X_{2}$ is nonempty. Since $\mathcal{G}^{k}$ is smooth, the only possibility is that $X_{1}=X_{2}$.

Therefore, to conclude, it will suffice to show that the restriction of $p$ to any component $X$ of $\mathcal{G}^{k}$ is dominant. To see this, let $(\widetilde{V}, L, \Lambda)$ be a point of $X$. By Proposition 3.12(2) applied to locus (4.4), we have 


$$
\operatorname{dim}_{(\widetilde{V}, L, \Lambda)}\left(\left.p\right|_{X}\right)^{-1}(p(\widetilde{V}, L, \Lambda)) \leq g-k(k-d+r(g-1))=\rho_{1,0, r, d}^{k} .
$$

On the other hand, by (4.3), we have

$$
\operatorname{dim}_{(\widetilde{V}, L, \Lambda)} X=\operatorname{dim} \mathcal{M}+\rho_{1,0, r, d}^{k} .
$$

Thus $\operatorname{dim}(p(X)) \geq \operatorname{dim}(\mathcal{M})$. As $\mathcal{M}$ is irreducible, $p(X)$ is dense in $\mathcal{M}$. This completes the proof.

\section{Nonemptiness of $B_{n, e}^{k}(V)$ and $\widetilde{B}_{n, e}^{k}(V)$}

We now prove Theorem 1.1 using the method of [24]. This is very straightforward; the necessary ingredients already exist by Theorem 2.1 and the results in [24] on stability of elementary transformations. We note that Mercat's construction was used in a similar way in $[3$, Sect. 6] to show the nonemptiness of certain moduli spaces of coherent systems.

Proof of Theorem 1.1 By hypothesis and Theorem 2.1, the locus $B_{1, e_{0}}^{k_{0}}(V)$ is of positive dimension. Thus we can find mutually nonisomorphic line bundles $L_{1}, \ldots, L_{n}$ of degree $e_{0}$ such that $h^{0}\left(V \otimes L_{i}\right) \geq k_{0}$ for each $i$. Let

$$
0 \rightarrow \bigoplus_{i=1}^{n} L_{i} \rightarrow E \rightarrow \tau \rightarrow 0
$$

be a general elementary transformation. We have the cohomology sequence

$$
0 \rightarrow \bigoplus_{i=1}^{n} H^{0}\left(V \otimes L_{i}\right) \rightarrow H^{0}(V \otimes E) \rightarrow \cdots
$$

so $h^{0}(V \otimes E) \geq n k_{0}$. If $\operatorname{deg} \tau \geq 1$, then it follows easily from [24, Théorème A.5] that $E$ is a stable vector bundle, and the result follows for $e>n e_{0}$. If $\tau=0$ then $E=\bigoplus_{i=1}^{n} L_{i}$ gives an element of $\widetilde{B}_{n, n e_{0}}^{n k_{0}}$, and the result follows also in the case $e=n e_{0}$.

In the next sections, we will refine this statement in some cases.

\section{Generatedness of Petri general bundles}

To prove the existence of components of $B_{n, e}^{k}(V)$ which are generically smooth and of the expected dimension, the need will emerge to show the existence of bundles $W$ of $\operatorname{rank} r \geq 2$ and degree $d$ satisfying the following conditions:

(1) $W$ is Petri trace injective. Equivalently, $K_{C} \otimes W^{*}$ is Petri trace injective.

(2) $h^{0}(W)=k \geq 1$.

(3) $K_{C} \otimes W^{*}$ is generically generated. 
Remark 6.1 Note that the above conditions give strong bounds on $d$ and $k$. By (1) and (2) and Serre duality, we have $k(k-d+r(g-1)) \leq g$, so $d \geq r(g-1)+k-\frac{g}{k}$. Moreover, (3) implies that $h^{0}\left(K_{C} \otimes W^{*}\right)=h^{1}(W)=k-\chi(W) \geq r$, so $d \leq r(g-2)+k$. In summary,

$$
r(g-1)+k-\frac{g}{k} \leq d \leq r(g-2)+k .
$$

Values of $d$ satisfying (6.1) exist if and only if $k \leq \frac{g}{r}$, which is in any case a necessary condition for (1) and (3) to hold.

In [15], Hitching and Hoff study the geometry of $B_{1, g-1}^{r+1}(V)$ for stable vector bundles of rank $r$ and vanishing determinant. Therefore, they also construct vector bundles with properties (1)-(3) (see [15, Prop. 2.8] and Proposition 6.5 as its generalisation).

\subsection{The construction}

Suppose $g \geq r$ and write $g=r l+r_{0}$ where $l, r_{0}$ are integers with $0 \leq r_{0}<r$. Let $D_{0}$ be an effective divisor of degree $r_{0}$ such that $h^{0}\left(K_{C}\left(-D_{0}\right)\right)=h^{0}\left(K_{C}\right)-r_{0}=r l$. (If $r_{0}=0$, take $D_{0}=0$.) Set $N:=K_{C}\left(-D_{0}\right)$ and let $D_{1}, \ldots, D_{r}$ be distinct effective divisors of degree $l$ such that

$$
h^{0}\left(N\left(-\sum D_{j}\right)\right)=h^{0}(N)-r l=0
$$

For $1 \leq i \leq r$, set $M_{i}:=N\left(-\sum_{j \neq i} D_{j}\right)$. By (6.2), we have $h^{0}\left(M_{i}\right)=h^{0}(N)-(r-1) l=l$ for each $i$.

\section{Lemma 6.2}

(1) $H^{0}(N) \cong \bigoplus_{i=1}^{r} H^{0}\left(M_{i}\right)$.

(2) $H^{1}\left(M_{i}\right) \cong H^{1}(N)=\mathbb{K}$.

(3) The bundles $M_{1}, \ldots, M_{r}$ are mutually nonisomorphic.

\section{Proof}

(1) We have inclusions $H^{0}\left(M_{i}\right) \hookrightarrow H^{0}(N)$ for all $i$. It is easy to see that

$$
H^{0}\left(M_{i}\right) \cap\left(H^{0}\left(M_{1}\right)+\cdots+H^{0}\left(M_{i-1}\right)+H^{0}\left(M_{i+1}\right)+\cdots+H^{0}\left(M_{r}\right)\right)=0 .
$$

So $\bigoplus_{i=1}^{r} H^{0}\left(M_{i}\right) \subseteq H^{0}(N)$. Since the dimensions agree, we obtain (1).

(2) Calculating values for degrees and $h^{0}$, this follows from Riemann-Roch.

(3) Suppose $M_{i_{1}} \cong M_{i_{2}}$; that is,

$$
N\left(-\sum_{j \neq i_{1}} D_{j}\right) \cong N\left(-\sum_{j \neq i_{2}} D_{j}\right) .
$$

Tensoring both sides with $N^{-1}\left(\sum_{j=1}^{r} D_{j}\right)$, we obtain $\mathcal{O}_{C}\left(D_{i_{1}}\right) \cong \mathcal{O}_{C}\left(D_{i_{2}}\right)$. As $D_{i_{1}} \neq D_{i_{2}}$ as divisors, in particular $h^{0}\left(\mathcal{O}_{C}\left(D_{i_{1}}\right)\right) \geq 2$. But then $h^{0}\left(K_{C}\left(-D_{i_{1}}\right)\right) \geq g-l+1$ and $h^{0}\left(M_{i_{2}}\right) \geq g-l+1-r_{0}-(r-2) l=l+1$, a contradiction. This proves (3). 
Now write $G:=\bigoplus_{i=1}^{r} M_{i}$. Consider elementary transformations

$$
0 \rightarrow W \rightarrow G \rightarrow T \rightarrow 0
$$

where $T$ is a torsion sheaf of degree $(r-1) l+m$ with $0 \leq m \leq l-1$. To ease notation, we write $t:=(r-1) l+m$. The set of such $W$ is parametrised by the Quot scheme $\operatorname{Quot}^{0, t}(G)$. By for example the proof of [11, Lemma 4.2], this is an irreducible variety of dimension $r t$.

Lemma 6.3 For general $W \in \operatorname{Quot}^{0, t}(G)$, the map $H^{0}(G) \rightarrow H^{0}(T)$ is surjective. In particular, $h^{0}(W)=l-m$ and $H^{0}\left(K_{C} \otimes W^{*}\right) \cong H^{0}\left(K_{C} \otimes G^{*}\right)$.

Proof Since the surjectivity condition is open, it is sufficient to prove the existence of one bundle $W$ with the required property. Suppose first that $t=1$ and let $T=\mathbb{K}_{p}$, where $p$ is a point at which some section of $G$ is nonzero. Then we can find a surjection $G \rightarrow T$ such that $H^{0}(G) \rightarrow H^{0}(T)$ is surjective. Repeating this argument, we obtain the result by induction on $t$.

We want one more generality condition on $W$. For $1 \leq i \leq r$, write $\hat{G}_{i}:=\bigoplus_{j \neq i} M_{j}$, and consider the sheaf

$$
W \cap \hat{G}_{i}=\operatorname{Ker}\left(W \rightarrow G \rightarrow M_{i}\right) .
$$

Lemma 6.4 If W is sufficiently general in $\operatorname{Quot}^{0, t}(G)$, then $h^{0}\left(W \cap \hat{G}_{i}\right)=0$.

Proof Since the condition $h^{0}\left(W \cap \hat{G}_{i}\right)=0$ is open, it is sufficient to find one example of an elementary transformation (6.3) for which this property holds. For this, consider elementary transformations

$$
0 \rightarrow W_{1} \rightarrow \hat{G}_{i} \rightarrow T \rightarrow 0
$$

where $T$ is as in (6.3). The same argument as for Lemma 6.3 shows that, for general $W_{1}$, we have $h^{0}\left(W_{1}\right)=\max \{0,-m\}=0$. Now take $W=W_{1} \oplus M_{i}$.

Proposition 6.5 A general elementary transformation $W \in \mathrm{Quot}^{0, t}(G)$ is stable and Petri trace injective and has $K_{C} \otimes W^{*}$ generically generated.

Proof As the $M_{i}$ are mutually nonisomorphic by Lemma 6.2(3), by [24, Théorème A.5] the bundle $W$ is stable for general $T$. By Lemma 6.3, we have $H^{0}\left(K_{C} \otimes W^{*}\right) \cong H^{0}\left(K_{C} \otimes G^{*}\right)$. From Lemma 6.2(2) and since $G=\oplus_{i=1}^{r} M_{i}$, it then follows that $K_{C} \otimes W^{*}$ is generically generated.

We describe $H^{0}\left(K_{C} \otimes W^{*}\right)$ more explicitly. For $1 \leq i \leq r$, let $t_{i}^{\prime}$ be a generator of $H^{0}\left(K_{C} \otimes M_{i}^{-1}\right)$, and write $t_{i}$ for the image of $t_{i}^{\prime}$ in $H^{0}\left(K_{C} \otimes W^{*}\right)$. Thus we obtain a splitting $H^{0}\left(K_{C} \otimes W^{*}\right)=\bigoplus_{i=1}^{r} \mathbb{K} \cdot t_{i}$. Therefore, we can write any element of $H^{0}(W) \otimes H^{0}\left(K_{C} \otimes W^{*}\right)$ in the form

$$
\sum_{i=1}^{r} \sigma_{i} \otimes t_{i}
$$


where $\sigma_{i}=\left(s_{i, 1}, s_{i, 2}, \ldots, s_{i, r}\right)$ is a section of $G$ belonging to $W$, that is, lying in the kernel of $H^{0}(G) \rightarrow H^{0}(T)$. The Petri trace is then given by

$$
\overline{\mu_{W}}\left(\sum_{i=1}^{r} \sigma_{i} \otimes t_{i}\right)=\sum_{i=1}^{r} \mu_{M_{i}}\left(s_{i, i} \otimes t_{i}^{\prime}\right) \in H^{0}\left(K_{C}\right) .
$$

To analyse this, note that, by Lemma 6.2(2), the homomorphism $M_{i} \rightarrow N$ induces an isomorphism $H^{0}\left(K_{C} \otimes N^{-1}\right) \rightarrow H^{0}\left(K_{C} \otimes M^{-1}\right)$. It follows that there is a commutative diagram

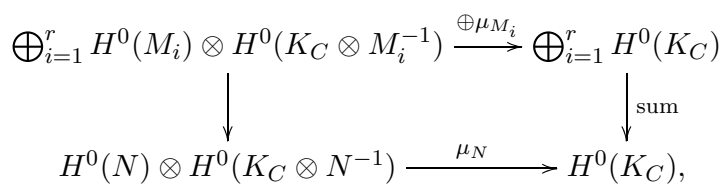

where the left-hand vertical map is an isomorphism by Lemma 6.2(1) and (2). Since $h^{0}\left(K_{C} \otimes N^{-1}\right)=1$ by Lemma 6.2(2), $\mu_{N}$ is injective. By commutativity, the composed map $\bigoplus_{i=1}^{r} H^{0}\left(M_{i}\right) \otimes H^{0}\left(K_{C} \otimes M_{i}^{-1}\right) \rightarrow H^{0}\left(K_{C}\right)$ is injective.

This means that a tensor of form (6.4) has trace zero only if $s_{i, i}=0$ for all $i$. Thus $\sigma_{i}$ belongs to the subsheaf $W \cap \hat{G}_{i}$. But by Lemma 6.4, for general $W \in \operatorname{Quot}^{0, t}(G)$, $h^{0}\left(W \cap \hat{G}_{i}\right)=0$ and $\sigma_{i}=0$ for all $i$. This completes the proof.

Corollary 6.6 Let $g, r, l=\left\lfloor\frac{g}{r}\right\rfloor$ and $r_{0}$ be as above. For $0 \leq m \leq l-1$, there exists a Petri trace injective bundle $W$ of rank $r$ and degree $r(g-2)+l-m$ with $h^{0}(W)=l-m$ and such that $K_{C} \otimes W^{*}$ is generically generated.

Corollary 6.7 Let $C$ be a general curve of genus $g$. Suppose $l=\left\lfloor\frac{g}{r}\right\rfloor, 0 \leq m \leq l-1$ and $d=r(g-2)+l-m$. If $m=0$, suppose further that $g \not \equiv 0 \bmod r$. Then $\left(B_{r, d}^{l-m}\right)_{\mathrm{PTI}}$ is irreducible and, if $W$ is a general element of $\left(B_{r, d}^{l-m}\right)_{\mathrm{PTI}}$ and $p$ is a general point of $C$,

$$
h^{0}(W)=h^{0}(W(p))=l-m .
$$

Proof The irreducibility of $\left(B_{r, d}^{l-m}\right)_{\mathrm{PTI}}$ follows from Theorem 4.3. (The hypothesis $g \not \equiv 0 \bmod r$ is required to ensure that the numerical hypothesis of Theorem 4.3 holds.) Property (6.5) is open. It is therefore sufficient to find one example of a bundle $W$ with the stated property. For this, take $W$ as in Corollary 6.6. Statement (6.5) is then equivalent to the injectivity of the coboundary map in the sequence

$$
\left.0 \rightarrow H^{0}(W) \rightarrow H^{0}(W(p)) \rightarrow W(p)\right|_{p} \rightarrow H^{1}(W) \rightarrow H^{1}(W(p)) \rightarrow 0 .
$$

By Serre duality, the coboundary map is injective if and only if the evaluation map

$$
\left.H^{0}\left(K_{C} \otimes W^{*}\right) \rightarrow K_{C} \otimes W^{*}\right|_{p}
$$

is surjective. By Corollary 6.6, this is true for general $p$. 


\section{Smoothness of twisted Brill-Noether loci}

In this section, we prove our main result Theorem 1.2. The major part of the section is concerned with proving the following technical proposition.

Proposition 7.1 Let $C$ be a general curve of genus $g$. Suppose $e_{0}$ and $k_{0}$ are integers satisfying

$$
\rho_{1, e_{0}, r, d}^{k_{0}}=g-k_{0}\left(k_{0}-\left(d+r e_{0}\right)+r(g-1)\right) \geq 1
$$

and furthermore that there exists a bundle $W \in\left(B_{r, d+r e_{0}}^{k_{0}}\right)_{\mathrm{PTI}}$ such that for general $p$ in $C$ we have $h^{0}(W)=h^{0}(W(p))=k_{0}$. Write $e=n e_{0}+e_{1}$ where $1 \leq e_{1} \leq n$. Then for general $V \in U^{s}(r, d)$ and for $r e+n d-r n(g-1) \leq k \leq n k_{0}$, the twisted Brill-Noether locus $B_{n, e}^{k}(V)$ has a component which is nonempty, generically smooth and of the expected dimension.

Note again that for $V \in U^{s}(r, d)$ and $E \in U^{s}(n, e)$, the Euler characteristic $\chi(V \otimes E)=r e+n d-r n(g-1)$. We begin with a lemma which has applications to coherent systems (twisted or untwisted) as well as to twisted Brill-Noether loci. For this, let $V$ be any bundle of rank $r$ and degree $d$ and consider the space $G^{k_{0}}\left(V, \mathcal{P}^{e_{0}}\right)$, where $\mathcal{P}^{e_{0}}$ is a Poincaré family on $\operatorname{Pic}^{e_{0}}(C) \times C$, which parametrises pairs $\left(L, \Lambda_{0}\right)$ with $L$ a line bundle of degree $e_{0}$ and $\Lambda_{0} \subset H^{0}(V \otimes L)$ a linear subspace of dimension $k_{0}$. Suppose further that $X$ is an irreducible component of $G^{k_{0}}\left(V, \mathcal{P}^{e_{0}}\right)$ which is generically smooth of dimension

$$
\rho_{1, e_{0}}^{k_{0}}(V)=g-k_{0}\left(k_{0}-\left(d+r e_{0}\right)+r(g-1)\right) \geq 1 .
$$

Let $\left(L_{1}, \Lambda_{1}\right), \ldots,\left(L_{n}, \Lambda_{n}\right)$ be points of $X$, and write $F:=\bigoplus_{i=1}^{n} L_{i}$ and $\Lambda:=\bigoplus_{i=1}^{n} \Lambda_{i}$. We consider elementary transformations

$$
0 \rightarrow F \rightarrow E \rightarrow \tau \rightarrow 0
$$

with $\tau$ a torsion sheaf of length $e_{1}$.

Lemma 7.2 Under the above conditions, let $\left(L_{1}, \Lambda_{1}\right), \ldots,\left(L_{n}, \Lambda_{n}\right)$ be general points of $X$. Then the restricted Petri E-trace map

$$
\mu_{E}: \Lambda \otimes H^{0}\left(K_{C} \otimes E^{*} \otimes V^{*}\right) \rightarrow H^{0}\left(K_{C} \otimes \text { End } E\right)
$$

is injective.

Proof Consider first the restricted Petri $F$-trace map of $V$, given by

$$
\mu_{F}: \Lambda \otimes H^{0}\left(K_{C} \otimes F^{*} \otimes V^{*}\right) \rightarrow H^{0}\left(K_{C} \otimes \text { End } F\right) .
$$

Noting that $H^{0}\left(K_{C} \otimes\right.$ End $\left.F\right)=\bigoplus_{i, j} H^{0}\left(K_{C} \otimes L_{j}^{-1} \otimes L_{i}\right)$, we see that (7.4) is the direct sum of the trace maps

$$
\mu_{i, j}: \Lambda_{i} \otimes H^{0}\left(K_{C} \otimes L_{j}^{-1} \otimes V^{*}\right) \rightarrow H^{0}\left(K_{C} \otimes L_{j}^{-1} \otimes L_{i}\right)
$$


for $1 \leq i \leq n$ and $1 \leq j \leq n$. Thus $\mu_{F}$ is injective if and only if $\mu_{i, j}$ is injective for all $i, j$.

Write

$$
U:=\left\{\left(L, \Lambda_{0}\right) \in X: X \text { smooth at }\left(L, \Lambda_{0}\right), h^{0}(V \otimes L) \text { takes its minimum value }\right\},
$$

which is a nonempty open subset of $X$ by semicontinuity. We can assume that $\left(L_{i}, \Lambda_{i}\right) \in U$ for all $i$.

Now let $p$ be a point of $C$. For each $\left(L, \Lambda_{0}\right) \in U$, we have a commutative diagram

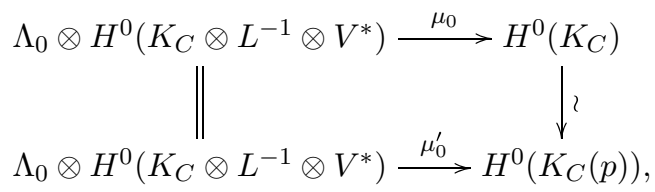

where, in the second line, $\Lambda_{0}$ is regarded as a subspace of $H^{0}(V \otimes L(p))$ and the horizontal arrows are trace maps. Since $X$ is smooth at $\left(L, \Lambda_{0}\right), \mu_{0}$ is injective. Hence so is $\mu_{0}^{\prime}$.

Next, let $A$ be the direct image sheaf over $U \times U$ whose fibre at $\left(\left(L, \Lambda_{0}\right),\left(N, \Lambda_{0}^{\prime}\right)\right)$ is

$$
\Lambda_{0} \otimes H^{0}\left(K_{C} \otimes N^{-1} \otimes V^{*}\right) .
$$

Since $H^{0}\left(K_{C} \otimes N^{-1} \otimes V^{*}\right)$ is constant on $U$, this is locally free. Furthermore, let $B$ be the direct image sheaf over $U$ whose fibre at $\left(\left(L, \Lambda_{0}\right),\left(N, \Lambda_{0}^{\prime}\right)\right)$ is $H^{0}\left(K_{C} \otimes N^{-1} \otimes L(p)\right)$. This is locally free of rank $g$.

Write $\tilde{\mu}: A \rightarrow B$ for the globalised Petri trace map whose restriction to $\left(\left(L, \Lambda_{0}\right),\left(N, \Lambda_{0}^{\prime}\right)\right)$ is the trace map

$$
\Lambda_{0} \otimes H^{0}\left(K_{C} \otimes N^{-1} \otimes V^{*}\right) \rightarrow H^{0}\left(K_{C} \otimes N^{-1} \otimes L(p)\right) .
$$

As $\left.\tilde{\mu}\right|_{\left(\left(L, \Lambda_{0}\right),\left(L, \Lambda_{0}\right)\right)}$ coincides with $\mu_{0}^{\prime}$ above, $\tilde{\mu}$ is injective on a nonempty open subset $U^{\prime}$ of $U \times U$. We can suppose that $\left(\left(L_{i}, \Lambda_{i}\right),\left(L_{j}, \Lambda_{j}\right)\right) \in U^{\prime}$ for all $i, j$, so that $\left.\tilde{\mu}\right|_{\left(\left(L_{i}, \Lambda_{i}\right),\left(L_{j}, \Lambda_{j}\right)\right)}$ is injective for all $i, j$. As $\left.\tilde{\mu}\right|_{\left(\left(L_{i}, \Lambda_{i}\right),\left(L_{j}, \Lambda_{j}\right)\right)}$ factors through the trace map (7.5), the latter is also injective. This completes the proof that $\mu_{F}$ is injective.

To see that $\mu_{E}$ is injective, we note that $K_{C} \otimes E^{*} \otimes V^{*} \subset K_{C} \otimes F^{*} \otimes V^{*}$ and consider the diagram of cohomology spaces

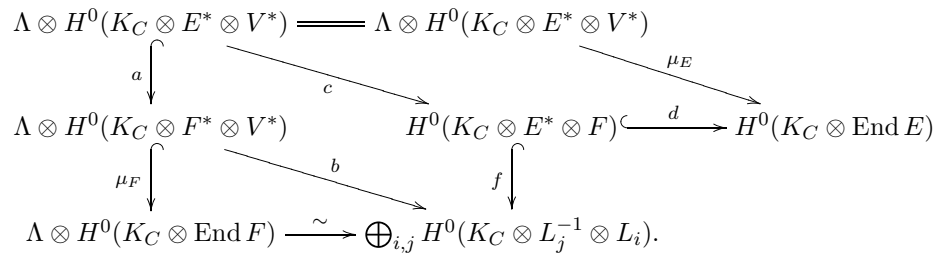

We have already seen that $\mu_{F}$ is injective. Hence first $b$, then $c$, then $\mu_{E}=d \circ c$ are all injective. 
Proof of Proposition 7.1 Suppose that the hypotheses of Proposition 7.1 hold and let $V \in U^{s}(r, d)$ be general. By Proposition 3.13, it suffices to exhibit a stable bundle $E \in U^{s}(n, e)$ with $h^{0}(V \otimes E)=n k_{0}$ and such that $V$ is Petri $E$-trace injective. For this, we use the construction of (7.2), where we now assume that this elementary transformation is general and the bundles $L_{i}$ are all distinct. It then follows from [24, Théorème A.5] that $E$ is stable.

Note next that, since $h^{0}(W(p))=k_{0}$, we must have $k_{0}>d+r e_{0}-r(g-1)$, so $\rho_{1, e_{0}}^{k_{0}}(V)<g$. By Theorem 2.1, it follows that the locus $B_{1, e_{0}}^{k_{0}}(V)$ is nonempty and irreducible of dimension $\rho_{1, e_{0}}^{k_{0}}(V) \geq 1$ (by (7.1)), and

$$
h^{0}(V \otimes L)=k_{0} \text { for general } L \in B_{1, e_{0}}^{k_{0}}(V) .
$$

By Theorem 3.9, we may assume also that $V$ is Petri general. By Theorem $4.3, V \otimes L$ is a general point of $\left(B_{r, d+r e_{0}}^{k_{0}}\right)_{\mathrm{PTI}}$. It now follows from the hypotheses of Proposition 7.1 that for general $p \in C$ we have

$$
h^{0}(V \otimes L(p))=k_{0} .
$$

Now let $L_{1}, \ldots, L_{n}$ be general points of $B_{1, e_{0}}^{k_{0}}(V)$, and write $F:=\bigoplus_{i=1}^{n} L_{i}$. We can assume that $h^{0}\left(V \otimes L_{i}\right)=k_{0}$ for all $i$, so that $h^{0}(V \otimes F)=n k_{0}$. Now note that the condition $h^{0}(V \otimes E)=n k_{0}$ is an open condition, so it is sufficient to exhibit a single elementary transformation (7.2) satisfying this condition. In fact, by (7.7), for general $p_{i} \in C$, we can take

$$
E_{0}:=\left(\bigoplus_{i=1}^{e_{1}} L_{i}\left(p_{i}\right)\right) \oplus\left(\bigoplus_{i=e_{1}+1}^{n} L_{i}\right) .
$$

Finally, we note that $B_{1, e_{0}}^{k_{0}}(V)$ is irreducible of the expected dimension by Theorem 2.1, and moreover $B_{1, e_{0}}^{k_{0}+1}(V)$ is of the expected dimension. It follows that $G^{k_{0}}\left(V, \mathcal{P}^{e_{0}}\right)$ is also irreducible of the expected dimension. Now apply Lemma 7.2 with $X=G^{k_{0}}\left(V, \mathcal{P}^{e_{0}}\right)$ and $\Lambda_{i}=H^{0}\left(V \otimes L_{i}\right)$ for all $i$. It follows that $V$ is Petri $E$-trace injective.

Now we can prove our main result on smoothness and dimension of twisted Brill-Noether loci.

Proof of Theorem 1.2 A straightforward computation shows that the numerical hypotheses and Corollary 6.7 imply that the hypotheses of Proposition 7.1 are satisfied.

\section{Remark 7.3}

(1) According to [32, Theorem 0.3], if $0 \leq \rho_{n, e}^{1}(V) \leq n^{2}(g-1)+1$, then every component of $B_{n, e}^{1}$ has dimension $\rho_{n, e}^{1}(V)$. The authors do not require the more stringent numerical conditions of our Theorem 1.2. Our result can be seen as a partial generalisation of [32, Theorem 0.3], although we do not show that every component of $B_{n, e}^{k}(V)$ has the expected dimension.

(2) It seems reasonable to conjecture that the hypotheses of Proposition 7.1 are satisfied in more cases than those covered in Theorem 1.2. The main obstacle to generalising 
the theorem is to show the generic generatedness of a general bundle in $\left(B_{r, d+r e_{0}}^{k_{0}}\right)_{\mathrm{PTI}}$ in more cases. (Theoretical bounds can be deduced from (6.1).)

Remark 7.4 For general $C$, the bundle $\mathcal{O}_{C}$ is Petri general and Petri trace injective. Moreover, when $r=1$, Corollary 6.7 holds without the restrictive numerical conditions. The above proofs are therefore valid, and we recover [10, Theorem 1.1] (see also [33]).

\section{Tangent cones of twisted Brill-Noether loci}

Suppose $Y \subset X$ are varieties, and let $x \in Y$ be a smooth point of $X$. Recall that the tangent

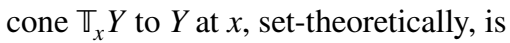

$$
\left\{v \in T_{x} X: v \text { is tangent to a smooth arc in } Y\right\} .
$$

Generalising theorems of Kempf [18] and Laszlo [21], in [9] the theory of determinantal varieties is used to describe the tangent cones to $B_{r, d}^{k}$ at points where the appropriate Petri maps are injective. In [9, Remark 2.8], it is noted that the same approach can be used to describe $\mathbb{T}_{E} B_{n, e}^{k}(V)$, which is a subvariety of $T_{E} U^{s}(n, e)=H^{1}($ End $E)$. In the following proposition, we follow up this remark and use Theorem 1.2 to give some situations in which it applies.

Proposition 8.1 Let $V \in U^{s}(r, d)$ and suppose that $E \in B_{n, e}^{k}(V)$ with $k \geq \chi:=\chi(V \otimes E)$ and $\mu_{E}$ injective.

(1) The tangent cone $\mathbb{T}_{E} B_{n, e}^{k}(V)$ is Cohen-Macaulay, reduced and normal.

(2) If $s_{1}, \ldots, s_{h^{0}(V \otimes E)}$ and $t_{1}, \ldots, t_{h^{1}(V \otimes E)}$ are bases for $H^{0}(V \otimes E)$ and $H^{0}\left(K_{C} \otimes E^{*} \otimes V^{*}\right)$ , respectively, then the ideal of $\mathbb{T}_{E} B_{n, e}^{k}(V)$ is generated by the minors of size $\left(h^{0}(V \otimes E)-k+1\right) \times\left(h^{0}(V \otimes E)-k+1\right)$ of the matrix whose $(i, j)$ th entry is

$$
\mu_{E}\left(s_{i} \otimes t_{j}\right)
$$

an element of $H^{0}\left(K_{C} \otimes\right.$ End $\left.E\right)=H^{1}(\text { End } E)^{*}$.

(3) The degree of $\mathbb{T}_{E} B_{n, e}^{k}(V)$ is

$$
\prod_{h=0}^{k-1} \frac{\left(h^{1}(V \otimes E)+h\right) ! \cdot h !}{\left(h^{0}(V \otimes E)-k+h\right) ! \cdot(k+h-\chi(V \otimes E)) !} .
$$

(4) As a set, $\mathbb{T}_{E} B_{n, e}^{k}(V)$ is the union

$$
\bigcup_{\Lambda \in \operatorname{Gr}\left(k, H^{0}(V \otimes E)\right)} \mu_{E}\left(\Lambda \otimes H^{0}\left(K_{C} \otimes E^{*} \otimes V^{*}\right)\right)^{\perp} \subseteq H^{1}(\text { End } E) .
$$

Proof If $k=\chi$, then $\mathbb{T}_{E} B_{n, e}^{k}(V)=H^{1}($ End $E)$, and the various parts of the proposition follow easily. (In particular, the formula in (3) yields the required degree 1.) Thus, in what follows, we will assume $k>\chi$.

As before, let $\mathcal{E} \rightarrow \widetilde{U^{s}(n, e)} \times C$ be a Poincaré bundle, where $\widetilde{U^{s}(n, e)} \rightarrow U^{s}(n, e)$ is a suitable étale cover. Fix a point in $\widetilde{U^{s}(n, e)}$ lying over $E$, and, abusing notation, denote 
it again by $E$. Recall the map $\pi: G^{k}(V, \mathcal{E})=: G_{n, e}^{k}(V) \rightarrow \widetilde{U^{s}(n, e)}$. By hypothesis, $\left.\mu_{E}\right|_{\Lambda \otimes H^{0}\left(K_{C} \otimes E^{*} \otimes V^{*}\right)}$ is injective for all $\Lambda \in \operatorname{Gr}\left(k, H^{0}(V \otimes E)\right)$. By Proposition 3.12(2), therefore, $G_{n, e}^{k}(V)$ is smooth and of dimension $\rho_{n, e}^{k}(V)$ in a neighbourhood of $\pi^{-1}(E)$, and $\pi^{-1}(E)$ is a smooth scheme. Moreover, by Proposition 3.13 the component of $B_{n, e}^{k}(V)$ containing $E$ also has dimension $\rho_{n, e}^{k}(V)$. As the fibres of $\pi$ are connected, being Grassmannians, $\pi$ is birational in a neighbourhood of $\pi^{-1}(E)$.

Thus the hypotheses of [1, II, Lemma 1.1 and Corollary, p. 66] are met, with $X$ being a suitable neighbourhood of $\pi^{-1}(E)$. Hence $\mathbb{T}_{E} B_{n, e}^{k}(V)=d \pi(N)$, where $N:=N_{\pi^{-1}(E) / G_{n, e}^{k}(V)}$ is the normal bundle of the fibre $\pi^{-1}(E)$ in $G_{n, e}^{k}(V)$.

Next, by Proposition 3.12(1) we have an exact sequence

$$
0 \rightarrow T_{\Lambda} \operatorname{Gr}\left(k, H^{0}(V \otimes E)\right) \rightarrow T_{(E, \Lambda)} G_{n, e}^{k}(V) \stackrel{d \pi}{\longrightarrow} T_{E} U^{s}(n, e)
$$

and

$$
\operatorname{Im}\left(\left.d \pi\right|_{(\Lambda, E)}\right)=\mu_{E}\left(\Lambda \otimes H^{0}\left(K_{C} \otimes E^{*} \otimes V^{*}\right)\right)^{\perp} \subseteq H^{1}(\text { End } E) .
$$

Thus the total space of the normal bundle $N$ can be identified with

$$
\left\{(\Lambda, v) \in \operatorname{Gr}\left(k, H^{0}(V \otimes E)\right) \times H^{1}(\text { End } E): v \cup \mu_{E}\left(\Lambda \otimes H^{0}\left(K_{C} \otimes E^{*} \otimes V^{*}\right)\right)=0\right\} .
$$

Moreover, via this identification, $d \pi$ is projection to the second factor.

This completes the proof of (4) and shows that the hypotheses of [1, Lemma p. 242] apply to $N$ (with $I=N, w=k, A=H^{0}(V \otimes E)$ and $\left.\phi=\mu_{E}\right)$. Therefore, statements (1-3) are, respectively, (i-iii) of [1, Lemma p. 242].

Remark 8.2 If the hypotheses of Theorem 1.2 apply, the conclusions of Proposition 8.1 hold for general $V$ and general $E \in B_{n, e}^{k}(V)_{0}$. In particular, we can describe some tangent cones of generalised theta divisors at well-behaved singular points. Suppose $g \geq r^{2}$ and $1 \leq d \leq r-1$. Then $\frac{g}{r} \geq r$, so we may set $k_{0}=d$. We write $r^{\prime}:=\frac{r}{\operatorname{gcd}(r, d)}$ and $d^{\prime}:=\frac{d}{\operatorname{gcd}(r, d)}$, and set $e_{0}=g-2$. Then for any positive integer $\lambda$, the values

$$
n=\lambda r^{\prime} \quad \text { and } \quad e=\lambda r^{\prime} e_{0}+\lambda\left(r^{\prime}-d^{\prime}\right)=\lambda \cdot\left(r^{\prime}(g-2)+r^{\prime}-d^{\prime}\right)
$$

satisfy both the hypotheses of Theorem 1.2 and the equation $r e+n d=r n(g-1)$. (Here $e_{1}:=e-n e_{0}=\lambda \cdot\left(r^{\prime}-d^{\prime}\right)$. Note also that necessarily $\left.n \geq 2\right)$.

By Theorem 1.2, for $1 \leq k \leq n k_{0}$ there exists a component $X_{k}$ of $B_{n, e}^{k}(V) \subset B_{n, e}^{1}(V)$ upon which the Petri maps $\mu_{E}$ are injective for general $E \in X_{k}$. By Proposition 8.1(3), for each such $E$ we have mult $_{E} B_{n, e}^{1}(V)=h^{0}(V \otimes E)$.

Geometry of the tangent cones We end this section with an observation generalising [9, Theorem 5.2] (see also [1, p. 232]). Firstly, we recall from [17, Sect. 3] that, generalising the canonical curve in $\left|K_{C}\right|^{*}$, for any vector bundle $E$ there is a map

$$
\mathbb{P} \text { End } E \rightarrow\left|\mathcal{O}_{\mathbb{P}\left(T_{C} \otimes \operatorname{End} E\right)}(1)\right|^{*} \cong \mathbb{P} H^{0}\left(K_{C} \otimes \text { End } E\right)^{*}=\mathbb{P} H^{1}(\text { End } E) .
$$

We write $\Delta$ for the closed sublocus $\mathbb{P} E^{*} \times_{C} \mathbb{P} E$ of rank one maps in $\mathbb{P}$ End $E$. Suppose $\phi_{1}, \ldots, \phi_{p}$ are points of $\Delta$ supported over distinct points $x_{1}, \ldots, x_{p}$ of $C$. For $1 \leq i \leq p$, let $\widetilde{\phi}_{i}$ be the point corresponding to $\phi_{i}$ via the identification 


$$
\text { End }\left.\left.E\right|_{x_{i}} \stackrel{\sim}{\rightarrow}(\text { End } E)\left(x_{i}\right)\right|_{x_{i}} \stackrel{\sim}{\rightarrow} H^{0}\left(\left.(\text { End } E)\left(x_{i}\right)\right|_{x_{i}}\right)
$$

which is canonical up to nonzero scalar. We observe that for any vector bundle $W$, the element $\widetilde{\phi}_{i}$ defines a map

$$
\left.\left.W \otimes E\right|_{x_{i}} \rightarrow W \otimes E\left(x_{i}\right)\right|_{x_{i}}
$$

This will be used later.

Now write $D=x_{1}+\cdots+x_{p}$. Unwinding the definition of map (8.1), we see that the secant $\overline{\phi_{1}, \ldots, \phi_{p}}$ in $\mathbb{P} H^{1}$ (End $E$ ) is the span of the images of the $\widetilde{\phi}_{i}$ by the coboundary map in the sequence

$$
0 \rightarrow H^{0}(\text { End } E) \rightarrow H^{0}((\text { End } E)(D)) \rightarrow H^{0}\left(\left.(\text { End } E)(D)\right|_{D}\right) \stackrel{\partial}{\rightarrow} H^{1}(\text { End } E) .
$$

The following is valid without any injectivity assumption on $\mu_{E}$.

Proposition 8.3 Let $m$ be the rank of the subbundle $V_{\text {ggen }}$ of $V$ generated by the evaluation map $E^{*} \otimes H^{0}(V \otimes E) \rightarrow V$. Suppose $h^{0}(V \otimes E) \geq p m+k$. Then $\operatorname{Sec}^{p} \Delta$ is contained in the projectivised tangent cone $\mathbb{P} \mathbb{T}_{E} B_{n, e}^{k}(V)$.

Proof (Compare with [9, Theorem 5.2]) A tangent vector $v \in H^{1}($ End $E$ ) belongs to $\mathbb{T}_{E} B_{n, e}^{k}(V)$ if and only if

$$
\operatorname{Ker}\left(\cdot \cup v: H^{0}(V \otimes E) \rightarrow H^{1}(V \otimes E)\right)
$$

has dimension at least $k$. Now clearly it suffices to show that a general point of $\operatorname{Sec}^{p} \Delta$ belongs to the tangent cone. So let $v=\partial\left(\lambda_{1} \widetilde{\phi}_{1}, \ldots, \lambda_{p} \widetilde{\phi}_{p}\right)$ where the $\widetilde{\phi}_{i}$ and $D$ are as above, and the $\lambda_{i}$ are nonzero scalars. The cup product map by $v$ factorises

$$
\left.\left.H^{0}(V \otimes E) \stackrel{\mathrm{ev}_{D}}{\longrightarrow} \bigoplus_{i=1}^{p} V \otimes E\right|_{x_{i}} \stackrel{\left(\lambda_{1} \widetilde{\phi}_{1}, \ldots, \lambda_{p} \widetilde{\phi}_{p}\right)}{\longrightarrow} \bigoplus_{i=1}^{p} V \otimes E\left(x_{i}\right)\right|_{x_{i}} \stackrel{\partial^{\prime}}{\longrightarrow} H^{1}(V \otimes E),
$$

where $\mathrm{ev}_{D}$ is the evaluation map, $\widetilde{\phi}_{i}$ is as in (8.2), and $\partial^{\prime}$ is the coboundary map of the cohomology sequence of

$$
\left.0 \rightarrow V \otimes E \rightarrow V \otimes E(D) \rightarrow V \otimes E(D)\right|_{D} \rightarrow 0 .
$$

Now $\operatorname{Im}\left(\mathrm{ev}_{D}\right)$ is contained in $\left.\bigoplus_{i=1}^{p} V_{\text {ggen }} \otimes E\right|_{x_{i}}$. In view of (8.2), moreover,

$$
\operatorname{Im}\left(\left(\lambda_{1} \tilde{\phi}_{1}, \ldots, \lambda_{p} \tilde{\phi}_{p}\right) \circ e v_{D}\right) \subseteq \bigoplus_{i=1}^{p} V_{\text {ggen }} \otimes \operatorname{Im}\left(\widetilde{\phi}_{i}\right) .
$$

Since by hypothesis $\operatorname{dim} \operatorname{Im}\left(\widetilde{\phi}_{i}\right)=1$, the last space has dimension $m p$. It follows that

$$
\operatorname{dim} \operatorname{Ker}(\cdot \cup v) \geq h^{0}(V \otimes E)-m p \geq k .
$$

The proposition follows.

The study of Petri-trace injective vector bundles leads naturally to the geometry of twisted Brill-Noether loci. We remark that a construction of Petri-trace injective bundles 
which are furthermore globally generated (similar as in Sect. 6) was used in [15] to prove the generic injectivity of the theta map.

\section{A nonempty twisted Brill-Noether locus with negative expected dimension}

It is well known that higher-rank Brill-Noether loci $B_{n, e}^{k}$ can exhibit more complicated behaviour than their rank one counterparts. Here we give an example of a nonempty twisted Brill-Noether locus with negative Brill-Noether number, where the curve $C$ and the bundle $V$ are general. Firstly, we recall some facts about maximal line subbundles of vector bundles (see [27] for more general and detailed information):

Let $C$ be a general curve of genus $g \geq 3$. Suppose $r \mid(g-1)$, and set $e_{0}:=(r-1) \frac{g-1}{r}$. Let $V$ be a general bundle of rank $r$ and degree zero over $C$. A computation shows that $\rho_{1, e_{0}}^{1}(V)=0$. As $V$ is general, by Theorem 2.1 (3) the locus $B_{1, e_{0}}^{1}(V)$ is of dimension zero. Furthermore, for $e<e_{0}$ or $k_{0}>1$ we check that $\rho_{1, e_{0}}^{k_{0}}(V)<0$. Hence, for all $L \in B_{1, e_{0}}^{1}(V)$, we have $h^{0}(V \otimes L)=1$ and $L^{-1}$ is a line subbundle of maximal degree in $V$. By [27, Proposition 1.4 and Lemma 2.2], $B_{1, e_{0}}^{1}(V)$ consists of $r^{g}$ points (of multiplicity 1).

Proposition 9.1 Let $C, r, g$ and $e_{0}$ be as above, and let $V$ be a general bundle of rank $r \geq 2$ and degree 0 . Let $n$ be an integer satisfying $r<n \leq r^{g}$. Then the twisted BrillNoether locus $B_{n, n e_{0}+1}^{n}(V)$ has negative expected dimension $n(r-n)+1$ but contains a component of dimension at least 1.

Remark 9.2 The construction below does not require $C$ and $V$ to be general. The hypothesis of generality is made so that the "expected dimension" makes sense.

Proof By the previous paragraph we can choose mutually nonisomorphic $L_{1}, \ldots, L_{n} \in B_{1, e_{0}}^{1}(V)$. Let

$$
0 \rightarrow \bigoplus_{i=1}^{n} L_{i} \rightarrow E \rightarrow \mathbb{K}_{p} \rightarrow 0
$$

be a general elementary transformation, where $\mathbb{K}_{p}$ is the skyscraper sheaf of degree 1 supported at $p \in C$. Then $E$ is stable by [24, Théorème A.5], and $h^{0}(V \otimes E) \geq n$. The Quot scheme parametrising the elementary transformations $E$ has dimension $n$; after acting by $\operatorname{Aut}\left(\oplus_{i=1}^{n} L_{i}\right)$, we see that there is precisely one stable $E$ for any given $p$. Thus $\operatorname{dim} B_{n e_{0}+1}^{n}(V) \geq 1$. On the other hand, we compute easily that

$$
\rho_{n, n e_{0}+1}^{n}(V)=1-n(n-r) .
$$

Since by hypothesis $n>r \geq 2$, this is negative. The result follows.

In exactly the same way, one can prove

Proposition 9.3 Let $C$ be a general curve and $V$ a bundle of rank $r \geq 2$ and degree $d$ over C. Suppose $k_{0} \geq 1$ and $e_{0}$ are integers satisfying $\rho_{1, e_{0}}^{k_{0}}(V)=0$. Assume that 


$$
\#\left(B_{1, e_{0}}^{k_{0}}(V)\right)>k_{0} r
$$

Then for $r k_{0}<n \leq \#\left(B_{1, e_{0}}^{k_{0}}(V)\right)$, the twisted Brill-Noether locus $B_{n, n e_{0}+1}^{n k_{0}}(V)$ is nonempty and has negative expected dimension $1-n\left(n-r k_{0}\right)$.

This example shows that even for general $C$ and general stable $V$, the twisted Brill-Noether loci can exhibit pathologies.

Acknowledgements Open Access funding provided by Projekt DEAL. We thank André Hirschowitz for answering several questions on rank stratifications. We also thank the referee for some useful comments.

Open Access This article is licensed under a Creative Commons Attribution 4.0 International License, which permits use, sharing, adaptation, distribution and reproduction in any medium or format, as long as you give appropriate credit to the original author(s) and the source, provide a link to the Creative Commons licence, and indicate if changes were made. The images or other third party material in this article are included in the article's Creative Commons licence, unless indicated otherwise in a credit line to the material. If material is not included in the article's Creative Commons licence and your intended use is not permitted by statutory regulation or exceeds the permitted use, you will need to obtain permission directly from the copyright holder. To view a copy of this licence, visit http://creativecommons.org/licenses/by/4.0/.

\section{References}

1. Arbarello, E., Cornalba, M., Griffiths, P.A., Harris, J.: Geometry of algebraic curves. In: Vol. I, Volume 267 of Grundlehren der Mathematischen Wissenschaften. Springer, New York (1985)

2. Beauville, A.: Vector bundles on curves and theta functions. In: Moduli Spaces and Arithmetic Geometry. Papers of the 13th International Research Institute of the Mathematical Society of Japan, Kyoto, Japan, September 8-15, 2004, pp. 145-156. Mathematical Society of Japan, Tokyo (2006)

3. Bhosle, U.N., Brambila-Paz, L., Newstead, P.E.: On coherent systems of type $(n, d, n+1)$ on Petri curves. Manuscr. Math. 126(4), 409-441 (2008)

4. Bradlow, S.B.: Coherent systems: a brief survey. with an appendix by H. Lange. In: Moduli Spaces and Vector Bundles. A tribute to Peter Newstead, pp. 229-264. Cambridge University Press, Cambridge (2009)

5. Bradlow, S.B., García-Prada, O., Muñoz, V., Newstead, P.E.: Coherent systems and Brill-Noether theory. Int. J. Math. 14(7), 683-733 (2003)

6. Brambila-Paz, L., Grzegorczyk, I.M., Newstead, P.E.: Geography of Brill-Noether loci for small slopes. J. Algebr. Geom. 6(4), 645-669 (1997)

7. Brambila-Paz, L., Lange, H.: A stratification of the moduli space of vector bundles on curves. J. Reine Angew. Math. 494, 173-187 (1998). Dedicated to Martin Kneser on the occasion of his 70th birthday

8. Brivio, S.: A note on theta divisors of stable bundles. Rev. Mat. Iberoam. 31(2), 601-608 (2015)

9. Casalaina Martin, S., Teixidor i Bigas, M.: Singularities of Brill-Noether loci for vector bundles on a curve. Math. Nachr. 284(14-15), 1846-1871 (2011)

10. Castorena, A., López Martín, A., Teixidor i Bigas, M.: Petri map for vector bundles near good bundles. J. Pure Appl. Algebra 222(7), 1692-1703 (2018)

11. Choe, I., Hitching, G.H.: Secant varieties and Hirschowitz bound on vector bundles over a curve. Manuscr. Math. 133(3-4), 465-477 (2010)

12. Ghione, F.: Un problème du type Brill-Noether pour les fibrés vectoriels. In: Algebraic GeometryOpen Problems (Ravello, 1982), Volume 997 of Lecture Notes in Mathematics, pp. 197-209. Springer, Berlin (1983)

13. Grzegorczyk, I.M., Teixidor i Bigas, M.: Brill-Noether theory for stable vector bundles. In: Moduli Spaces and Vector Bundles, Volume 359 of London Mathematical Society. Lecture Note Series, pp. 29-50. Cambridge University Press, Cambridge (2009)

14. Hirschowitz, A.: Problèmes de Brill-Noether en rang supérieur. C. R. Acad. Sci. Paris Sér. I Math. 307(4), 153-156 (1988)

15. Hitching, G.H., Hoff, M.: Tangent cones to generalised theta divisors and generic injectivity of the theta map. Compos. Math. 153(4), 2643-2657 (2017) 
16. Holla, Y.I.: Counting maximal subbundles via Gromov-Witten invariants. Math. Ann. 328(1-2), 121133 (2004)

17. Hwang, J.-M., Ramanan, S.: Hecke curves and Hitchin discriminant. Ann. Sci. Éc. Norm. Supér. 37(4), 801-817 (2004)

18. Kempf, G.R.: On the geometry of a theorem of Riemann. Ann. Math. 98(2), 178-185 (1973)

19. Lange, H., Narasimhan, M.S.: Maximal subbundles of rank two vector bundles on curves. J. Reine Angew. Math. 266, 55-72 (1983)

20. Lange, H., Newstead, P.E.: Maximal subbundles and Gromov-Witten invariants. In: A Tribute to C. S. Seshadri. Birkhäuser, Basel (2003)

21. Laszlo, Y.: Un théorème de Riemann pour les diviseurs thêta sur les espaces de modules de fibrés stables sur une courbe. Duke Math. J. 64(2), 333-347 (1991)

22. Lazarsfeld, R.: Some applications of the theory of positive vector bundles. In: Complete Intersections (Acireale, 1983), Volume 1092 of Lecture Notes in Mathematical, pp. 29-61. Springer, Berlin (1984)

23. Mercat, V.: Le problème de Brill-Noether pour des fibrés stables de petite pente. J. Reine Angew. Math. 506, 1-41 (1999)

24. Mercat, V.: Le problème de Brill-Noether et le théorème de Teixidor. Manuscr. Math. 98(1), 75-85 (1999)

25. Narasimhan, M.S., Ramanan, S.: Deformations of the moduli space of vector bundles over an algebraic curve. Ann. Math. 2(101), 391-417 (1975)

26. Newstead, P.E.: Existence of $\alpha$-stable coherent systems on algebraic curves. In: Grassmannians, Moduli Spaces and Vector Bundles, Volume 14 of Clay Mathematics Proceedings, pp. 121-139. American Mathematical Society, Providence, RI (2011)

27. Oxbury, W.M.: Varieties of maximal line subbundles. Math. Proc. Camb. Philos. Soc. 129(1), 9-18 (2000)

28. Pauly, C.: On the base locus of the linear system of generalized theta functions. Math. Res. Lett. 15(4), 699-703 (2008)

29. Popa, M.: On the base locus of the generalized theta divisor. C. R. Acad. Sci. Paris Sér. I Math. 329(6), 507-512 (1999)

30. Popa, M.: Generalized theta linear series on moduli spaces of vector bundles on curves. In: Handbook of Moduli. Vol. III, Volume 26 of Advanced Lectures in Mathematics (ALM), pp. 219-255. International Press, Somerville (2013)

31. Raynaud, M.: Sections des fibres vectoriels sur une courbe. Bull. Soc. Math. Fr. 110, 103-125 (1982)

32. Russo, B., Teixidor i Bigas, M.: On a conjecture of Lange. J. Algebr. Geom. 8(3), 483-496 (1999)

33. Teixidor i Bigas, M.: Brill-Noether theory for stable vector bundles. Duke Math. J. 62(2), 385-400 (1991)

34. Teixidor i Bigas, M.: Petri map for rank two bundles with canonical determinant. Compos. Math. 144(3), 705-720 (2008)

35. Teixidor i Bigas, M.: Injectivity of the Petri map for twisted Brill-Noether loci. Manuscr. Math. 145(3-4), 389-397 (2014)

36. Teixidor i Bigas, M., Tu, L.W.: Theta divisors for vector bundles. In: Curves, Jacobians, and Abelian Varieties (Amherst, MA, 1990), Volume 136 of Contemporary Mathematics, pp. 327-342. American Mathematical Society, Providence, RI (1992)

Publisher's Note Springer Nature remains neutral with regard to jurisdictional claims in published maps and institutional affiliations. 